\title{
Bifurcation from the essential spectrum
}

\author{
C. A. Stuart \\ March 4, 2001
}

\section{Contents}

1 Introduction 2

2 General setting $\quad 3$

$\begin{array}{lll}3 & \text { Nonlinear perturbation of a self-adjoint operator } & 8\end{array}$

4 Bifurcation from the infimum of the spectrum 12

4.1 The fixed $\lambda$ approach . . . . . . . . . . . 13

4.2 The fixed norm approach . . . . . . . . . . 21

$\begin{array}{lll}\mathbf{5} & \text { Bifurcation into spectral gaps } & \mathbf{3 0}\end{array}$

$\begin{array}{lll}6 & \text { Semilinear elliptic equations } & \mathbf{4 0}\end{array}$ 


\section{Introduction}

This report surveys some of the results that have been obtained during the past twenty years concerning bifurcation from a point in the essential spectrum of the linearization of a nonlinear equation. This means that the linearization is not a Fredholm operator and so, even locally, the problem cannot be reduced to an equivalent finite dimensional situation by the method of Lyapunov- Schmidt. Nonetheless, the aim is to obtain conclusions, local or global, about bifurcation in the same spirit as the classical results which are well-known in the Fredholm case. A vast survey of recent progress in bifurcation theory for the Fredholm situation has been given by Ize [30] in an article in the previous volume of this series. In the present context all of the standard techniques of nonlinear analysis (variational methods, topological degree, implicit function theorems) have been brought to bear on the problem, but so far only the variational approach has yielded general results in abstract spaces. The other methods have been confined to the context of elliptic equations on unbounded domains or to integral equations involving convolution. This state of affairs is reflected in the presentation of this survey which concentrates on the general results obtained by variational methods and their application to elliptic equations on $\Re^{N}$. However in the last section there are a few remarks covering what is known about connected sets, or even curves, of solutions to differential equations and it is to be hoped that in the near future substantial progress will be made in establishing conclusions of this kind in an abstract setting similar to that used in the variational case. 


\section{General setting}

The general results in this part of the survey concern nonlinear eigenvalue problems which have the following structure.

Let $(H,\langle\cdot, \cdot\rangle)$ be a real Hilbert space with norm $\|\cdot\|=\langle\cdot, \cdot\rangle^{1 / 2}$. The space of bounded linear operators on $H$ is denoted by $B(H, H)$ and, for $L \in B(H, H)$, the adjoint of $L$ is denoted by $L^{*}$. We consider the equation

$$
\nabla J(u)=\lambda L u \text { for }(\lambda, u) \in \Re \times H
$$

where the functions $J$ and $L$ have the following properties.

(H) $J \in C^{2}(H, \Re)$ with $J^{\prime}(0)=0$ and $L \in B(H, H)$ with $L=L^{*}$ and $\langle L u, u\rangle>0$ for all $u \in H \backslash\{0\}$.

In this context we can assume without loss of generality that $J(0)=0$. It is convenient to introduce some notation for the Taylor expansion of $J$ about 0 . The gradient, $\nabla J: H \longrightarrow H$, of $J$ is defined by

$$
\langle\nabla J(u), v\rangle=J^{\prime}(u) v \text { for } u, v \in H
$$

and there is an operator, $A \in B(H, H)$, such that

$$
\langle A u, v\rangle=J^{\prime \prime}(0)(u, v) \text { for } u, v \in H .
$$

It follows from $(\mathrm{H})$ that $A=A^{*}$ and, setting

$$
N(u)=A u-\nabla J(u) \text { and } \varphi(u)=1 / 2\langle A u, u\rangle-J(u),
$$

we have that

$$
\varphi \in C^{2}(H, \Re) \text { that } \nabla \varphi=N \text { and that } \lim _{\|u\| \rightarrow 0} \varphi(u) /\|u\|^{2}=0 .
$$

The equation (1) can now be expressed as

$$
A u-N(u)=\lambda L u \text { for }(\lambda, u) \in \Re \times H .
$$

Henceforth, we discuss the equation (2) under the following slightly less restrictive hypotheses. 
(H1) $A, L \in B(H, H)$ with $A=A^{*}, L=L^{*}$ and $\langle L u, u\rangle>0$ for all $u \in H \backslash\{0\}$

and

(H2) $\varphi \in C^{1}(H, \Re)$ with $\nabla \varphi=N$ and $\lim _{\|u\| \rightarrow 0} \varphi(u) /\|u\|^{2}=0$.

Without loss of generality we have supposed that $J(u)=\varphi(u)=0$. Clearly (H2) implies that $N(0)=0$, and that $N$ is Fréchet differentiable at 0 with $N^{\prime}(0)=0$.

Under the hypotheses (H1) and (H2), we set

$$
J(u)=1 / 2\langle A u, u\rangle-\varphi(u)
$$

and then the equation (2) can be written in the form of (1). The condition (H) is satisfied provided that, in addition to (H1) and (H2), we have $\varphi \in$ $C^{2}(H, \Re)$.

Henceforth we deal with the equation (2) under the hypotheses (H1) and (H2). In order to avoid the trivial solutions $(\lambda, 0)$ we set

$$
E=\{(\lambda, u) \in \Re \in H: A u-N(u)=\lambda L u \text { and } u \neq 0\}
$$

but we begin by investigating the set $E$ close to the line of trivial solutions.

Definition 2.1 The bifurcation points for the equation (2) are the elements of the set $B$ defined by

$$
B=\{\lambda \in \Re:(\lambda, 0) \in \bar{E}\}
$$

where $\bar{E}$ denotes the closure of $E$ in $\Re \times H$.

Thus $\lambda \in B$ if and only if there is a sequence of solutions $\left\{\left(\lambda_{n}, u_{n}\right)\right\}$ of (2) such that

$$
u_{n} \neq 0, \lim \left\|u_{n}\right\|=0 \text { and } \lim \lambda_{n}=\lambda .
$$

Clearly $B$ is a closed subset of $\Re$. If $\lambda_{n}<\lambda$ for all $n \in \aleph$ we say that there is bifurcation to the left at $\lambda$, and if in addition

$$
\lim _{n \rightarrow \infty}\left(\lambda-\lambda_{n}\right)^{-\theta}\left\|u_{n}\right\|=0
$$


we say that this bifurcation is of order $\theta$.

One expects to obtain some information about solutions of equation (2) with $\|u\|$ small by studying its first order approximation $A u=\lambda L u$ and this leads us to seek a relation between the set $B$ and the spectrum of $A$.

Definition 2.2 Under the hypothesis (H1), we define the resolvent set, $\rho(A, L)$, of $A$ with respect to $L$ by

$$
\rho(A, L)=\{\lambda \in \Re: A-\lambda L: H \longrightarrow H \text { is an isomorphism }\}
$$

and then the spectrum, $\sigma(A, L)$, of $A$ with respect to $L$ by $\sigma(A, L)=\Re \backslash$ $\rho(A, L)$.

The discrete spectrum, $\sigma_{d}(A, L)$, of $A$ is defined by

$$
\sigma_{d}(A, L)=\{\lambda \in \sigma(A, L): A-\lambda L: H \longrightarrow H \text { is a Fredholm operator }\}
$$

and then the essential spectrum, $\sigma_{e}(A, L)$, of $A$ by $\sigma_{e}(A, L)=\sigma(A, L) \backslash$ $\sigma_{d}(A, L)$.

In the case where $L=I$ we write $\sigma(A)$ for $\sigma(A, I)$ etc. and we have the following well-known properties of these sets.

1. For $\lambda \in \rho(A), A-\lambda I: H \longrightarrow H$ is a homeomorphism.

2. The spectrum, $\sigma(A)$, is a non-empty closed subset of $\Re$ and $\sigma_{e}(A)$ is a closed subset of $\sigma(A)$.

3. The spectral theorem shows that $\lambda \in \sigma_{d}(A)$ if and only if $\lambda$ is an isolated (with respect to $\sigma(A)$ ) eigenvalue of finite multiplicity of $A$.

4. The essential spectrum cannot be changed by a compact perturbation in the sense that $\sigma_{e}(A+C)=\sigma_{e}(A)$ for any compact linear operator $C: H \longrightarrow H$.

The fundamental relationship between the discrete spectrum of $A$ with respect to $L$ and the set of bifurcation points for equation (2) is established by a famous classical result due to Krasnoselskii, and later improved in several ways [35], [6], [50], [53], [66], [46], [51]. 
Theorem 2.3 Let the hypotheses (H1) and (H2) be satisfied. Then

$$
\overline{\sigma_{d}(A, L)} \subset B \subset \sigma(A, L) .
$$

Remark 1 If $\operatorname{dim} H<\infty$, we have that $\sigma_{d}(A, L)=\sigma(A, L)$ and consequently that $B=\sigma(A, L)$.

Remark 2 If $\operatorname{dim} H=\infty$, if $A$ is compact and if $L$ is positive definite, we have that $\sigma(A, L)=\sigma_{d}(A, L) \cup\{0\}$ and so the above result still covers all but one point in $\sigma(A, L)$. However, if $A$ is not compact it may be that $\sigma_{d}(A, L)=\emptyset$ even for $L=I$, and in such cases the above result gives no information about the existence of bifurcation points for equation (2). The results in this survey go some way towards completing this picture.

Let us end these introductory remarks by recalling three well-known examples of self-adjoint operators having no discrete spectrum.

Example 1 Let $H=L^{2}([0,1])$ with $\langle u, v\rangle=\int_{0}^{1} u(x) v(x) d x$ and consider a bounded measurable function $f:[0,1] \longrightarrow \Re$. The multiplication operator, $A$, defined by

$$
(A u)(x)=f(x) u(x) \text { for } x \in[0,1]
$$

together with $L=I$ satisfy the condition (H1) and $\sigma(A)=\sigma_{e}(A)=\overline{R(f)}$ where $\overline{R(f)}$ denotes the closure of the range of $f$.

Nonlinear eigenvalue problems involving this operator have been discussed in [43], [44]. They provide simple explicitly solvable examples which shed some light on the precision of the general results.

Example 2 Let $H=L^{2}(\Re)$ with $\langle u, v\rangle=\int_{-\infty}^{\infty} u(x) v(x) d x$ and consider the convolution integral operator defined by

$$
(A u)(x)=\int_{-\infty}^{\infty} k(x-y) u(y) d y \text { for } x \in \Re
$$

where $k \in L^{1}(\Re)$. This operator and $L=I$ satisfy the condition (H1) and $\sigma(A)=\sigma_{e}(A)=\overline{R(\hat{k})}$ where $\hat{k}$ denotes the Fourier transform of $k$.

Nonlinear equations involving this kind of operator have been treated in [19], [55]. They arise in models for the liquid-gas interface and in plasma physics. 
Example 3 Let $H$ denote the Sobolev space $H^{1}\left(\Re^{N}\right)$ with

$$
\langle u, v\rangle=\int_{\Re^{N}}\{u(x) v(x)+\nabla u(x) . \nabla v(x)\} d x .
$$

Let $V: \Re^{N} \longrightarrow \Re$ be a bounded measurable function which is periodic in the sense that $V(x+k)=V(x)$ for all $x \in \Re^{N}$ and all $k \in Z^{N}$. Two bounded self-adjoint operators $A$ and $L$ are defined by the relations

$$
\langle A u, v\rangle=\int_{\Re^{N}}\{V(x) u(x) v(x)+\nabla u(x) \cdot \nabla v(x)\} d x
$$

and

$$
\langle L u, v\rangle=\int_{\Re^{N}} u(x) v(x) d x \text { for all } u, v \in H .
$$

To show that $\sigma_{d}(A, L)=\emptyset$ we consider the complexification $\tilde{H}$ of $H$ and translation operator $T: \tilde{H} \longrightarrow \tilde{H}$ defined by $(T u)(x)=u\left(x+e_{1}\right)$ for $x \in \Re^{N}$ where $e_{1}=(1,0, \ldots, 0)$. Clearly $T$ is a unitary operator and, for any $\lambda \in \Re, T$ leaves $\operatorname{ker}(A-\lambda L)$ invariant. Hence if $0<\operatorname{dim} \operatorname{ker}(A-\lambda L)<\infty$, there exist $\mu \in C$ and $w \in \operatorname{ker}(A-\lambda L) \backslash\{0\}$ such that $|\mu|=1$ and $T w=\mu w$. But then $\left|w\left(x+e_{1}\right)\right|=|w(x)|$ for all $x \in \Re^{N}$ and this is incompatible with the fact that $w \in \tilde{H} \backslash\{0\}$. Hence either $\operatorname{ker}(A-\lambda L)=\{0\}$ or $\operatorname{dim} \operatorname{ker}(A-\lambda L)=\infty$. In the latter case it is easy to deduce that $\operatorname{dim}[\operatorname{ker}(A-\lambda L) \cap H]=\infty$ too. It follows that $\sigma_{d}(A, L)=\emptyset$.

The operators $A$ and $L$ in this example are used in the weak formulation of nonlinear perturbations of the Schrödinger operator, $-\Delta u(x)+V(x) u(x)$, with a periodic potential, $V$. Equations of this kind arise in various fields such as nonlinear optics and solid state physics [78], [13]. Some of the results obtained by variational methods concerning bifurcation for the equation

$$
-\Delta u(x)+V(x) u(x)+f(x, u(x))=\lambda u(x) \text { for } x \in \Re^{N}
$$

where $f(x, 0) \equiv \partial_{u} f(x, 0) \equiv 0$, are summarized in Section 6 of this report. 


\section{Nonlinear perturbation of a self-adjoint op- erator}

Many nonlinear eigenvalue problems involving differential equations appear as nonlinear perturbations of an unbounded self-adjoint operator, $S$. In this section we give conditions under which such problems can be cast in the form (2) with $\sigma(A, L)=\sigma(S)$.

Let $(\mathcal{H},(\cdot, \cdot))$ be a real Hilbert space and consider a self-adjoint operator $S: D(S) \subset \mathcal{H} \longrightarrow \mathcal{H}$ such that $\sigma(S) \neq \Re$. Our purpose is to discuss an equation of the form

$$
S u+R(u)=\lambda u \text { for }(\lambda, u) \in \Re \times[D(S) \cap D(R)]
$$

where $R: D(R) \subset \mathcal{H} \longrightarrow \mathcal{H}$ is a nonlinear operator whose properties will be defined later.

We introduce the Hilbert space $(H,\langle\cdot, \cdot\rangle)$ defined by $H=D\left(|S|^{1 / 2}\right)$ and

$$
\langle u, v\rangle=(u, v)+\left(|S|^{1 / 2} u,|S|^{1 / 2} v\right) \text { for } u, v \in D\left(|S|^{1 / 2}\right) \text {. }
$$

Then $\|u\|=<u, u>^{1 / 2}$ is the graph norm of $|S|^{1 / 2}$ and we recall that $D(S)$ is a dense subset of $H$ for this norm. In the terminology of [21], page 183, $H$ is called the form domain of $S$, and so following this lead we refer to $(H,\langle\cdot, \cdot\rangle)$ as the form space of $S$.

It is easy to check that, for $u \in D(S)$ and $v \in H$,

$$
|(S u, v)| \leq\|u\| \quad\|v\|
$$

Hence there is a unique continuous bilinear form $B: H \times H \longrightarrow \Re$ such that $B(u, v)=(S u, v)$ for all $u \in D(S)$ and $v \in H$; and there is a unique operator $A \in B(H, H)$ such that

$$
A=A^{*} \text { and }\langle A u, v\rangle=B(u, v) \text { for all } u, v \in H .
$$

Also there is a unique operator $L \in B(H, H)$ such that

$$
\langle L u, v\rangle=(u, v) \text { for all } u, v \in H .
$$


Clearly $L=L^{*}$ and $\langle L u, u\rangle=|u|^{2}>0$ for all $u \in H \backslash\{0\}$.

Noting that $\sigma(S-\mu I)=\sigma(S)-\mu$ and that $\sigma(A-\mu L, L)=\sigma(A, L)-\mu$ for all $\mu \in \Re$, it is easy to deduce from the results in Section 8 of [79] that

$$
A L=L A \text { and that } \sigma(S)=\sigma(A, L) .
$$

Next we consider a nonlinear operator $R: D(R) \subset \mathcal{H} \longrightarrow \mathcal{H}$ with $D(R)=$ $D(S)$. Then every solution of $S u+R(u)=\lambda u$ satisfies the equation $A u+$ $\tilde{L} R(u)=\lambda L u$ where $\tilde{L} \in B(\mathcal{H}, H)$ is such that

$$
\langle\tilde{L} u, v\rangle=(u, v) \text { for all } v \in H .
$$

Thus, if we suppose that the functional $\varphi$ in (H2) can be chosen in such a way that $N(u)=\tilde{L} R(u)$ for all $u \in D(S)$, it follows that every solution of (3) will also satisfy (2). Conversely if $(\lambda, u) \in \Re \times H$ satisfies (2) and $u \in D(S)$ then $(\lambda, u)$ is a solution of $(3)$.

We give two typical examples of this situation which will be used to illustrate the general results on bifurcation.

Example 1 Let $\mathcal{H}=L^{2}\left(\Re^{N}\right)$ with the usual norm. We consider squareintegrable solutions of the following elliptic equation

$$
-\Delta u(x)+V(x) u(x)-r(x)|u(x)|^{p-2} u(x)=\lambda u(x) \text { for } x \in \Re^{N}
$$

where

(A1) $V \in L^{\infty}\left(\Re^{N}\right)$

(A2) $r \in L^{\infty}\left(\Re^{N}\right)$ with $r \geq 0$ and $2<p<2^{*}$ where $2^{*}=\infty$ for $N=1$ and 2 and $2^{*}=2 N /(N-2)$ for $N \geq 3$.

Under the assumption (A1) it is well-known that a self-adjoint operator $S: D(S) \subset L^{2}\left(\Re^{N}\right) \longrightarrow L^{2}\left(\Re^{N}\right)$ is defined by

$$
S u(x)=-\Delta u(x)+V(x) u(x) \text { with } D(S)=H^{2}\left(\Re^{N}\right) .
$$

Furthermore $S$ is bounded below and the form space $(H,\langle\cdot, \cdot\rangle)$ of $\mathrm{S}$ coincides, up to equivalence of norms, with the Sobolev space $H^{1}\left(\Re^{N}\right)$.

Setting

$$
\varphi(u)=(1 / p) \int_{\Re^{N}} r(x)|u(x)|^{p} d x,
$$


the Sobolev embedding theorems imply that $\varphi \in C^{2}\left(H^{1}\left(\Re^{N}\right), \Re\right)$ and that $R(u):=r|u|^{p-2} u \in L^{2}\left(\Re^{N}\right)$ for all $u \in H^{2}\left(\Re^{N}\right)$. Since

$$
\varphi^{\prime}(u) v=\int_{\Re^{N}} r(x)|u(x)|^{p-2} u(x) v(x) d x \text { for all } u, v \in H^{1}\left(\Re^{N}\right)
$$

it follows that $N(u)=\tilde{L} R(u)$ for all $u \in H^{2}\left(\Re^{N}\right)$.

Thus, under the hypotheses (A1) and (A2), the equation (4) can be cast in the form (2) where the conditions (H1) and (H2) are satisfied and solutions of (2) correspond to weak solutions of (4) in the usual sense. Furthermore, solutions of (2) are in fact strong solutions of (4). As is shown in [29] this can be deduced from results due to Agmon [1] on elliptic regularity. Indeed, if $(\lambda, u) \in \Re \times H^{1}\left(\Re^{N}\right)$ is a solution of (2) then we find that $u \in L^{\infty}\left(\Re^{N}\right) \cap$ $H^{2}\left(\Re^{N}\right) \cap C^{1}\left(\Re^{N}\right)$ and that

$$
\lim _{|x| \rightarrow \infty}|u(x)|=0
$$

Example 2 Here we show how homoclinic solutions of a first order Hamiltonian system can be studied using an equation of the form (2). We consider the differential equation

$$
J u^{\prime}(t)+M u(t)-\|K(t) u(t)\|^{p-2} K(t)^{T} K(t) u(t)=\lambda u(t)
$$

and we seek solutions satisfying the condition

$$
\lim _{|t| \rightarrow \infty} u(t)=0
$$

In (7), $u(t) \in \Re^{2 N}$ and $J, M$ and $K(t)$ are real $(2 N \times 2 N)$-matrices. The transpose of a matrix $F$ is denoted by $F^{T}$. To treat (7) with (8), we make the following hypotheses.

(C1) $J=-J^{T}=-J^{-1}, M=M^{T}$ and the matrix $J M$ has no eigenvalues with real part equal to zero

(C2) $K \in L^{\infty}\left(\Re, M_{2 N}\right)$ and $p>2$ where $M_{2 N}$ denotes the space of real $(2 N \times 2 N)-$ matrices.

As the basic Hilbert space we choose $\mathcal{H}=\left[L^{2}(\Re)\right]^{2 N}$ with the usual scalar product. A self-adjoint operator, $S: D(S) \subset \mathcal{H} \longrightarrow \mathcal{H}$, is defined by

$$
D(S)=\left[H^{1}(\Re)\right]^{2 N} \text { and } S u(t)=J u^{\prime}(t)+M u(t) .
$$


We have shown in [79] that, up to equivalence of norms, the form space $(H,\langle\cdot, \cdot\rangle)$ of $S$, is equal to the fractional Sobolev space $\left[H^{1 / 2}(\Re)\right]^{2 N}$ whose scalar product is defined by

$$
\langle u, v\rangle=\int_{\Re}\left(1+|\xi|^{2}\right)^{1 / 2}[\hat{u}(\xi), \hat{v}(\xi)] d \xi
$$

where $[\cdot, \cdot]$ denotes the scalar product in $C^{2 N}$.

Setting

$$
\varphi(u)=(1 / p) \int_{\Re}|K(t) u(t)|^{p} d t \text { for } u \in\left[H^{1}(\Re)\right]^{2 N}
$$

we find that $\varphi \in C^{2}\left(\left[H^{1 / 2}(\Re)\right]^{2 N}, \Re\right)$ and that

$$
\varphi^{\prime}(u) v=\int_{\Re}\|K(t) u(t)\|^{p-2}[K(t) u(t), K(t) v(t)] d t .
$$

for all $u, v \in\left[H^{1 / 2}(\Re)\right]^{2 N}$.

It follows that $N(u)=\tilde{L} R(u)$ where

$R(u)(t)=\|K(t) u(t)\|^{p-2} K(t)^{T} K(t) u(t) \in\left[L^{2}(\Re)\right]^{2 N}$ for all $u \in\left[H^{1 / 2}(\Re)\right]^{2 N}$.

Hence the equation (7) can be expressed in the form (2) and, as is shown in Lemma 10.6 of [79], if $(\lambda, u) \in \Re \times\left[H^{1 / 2}(\Re)\right]^{2 N}$ is a solution of (2) then $u \in\left[H^{1}(\Re)\right]^{2 N}$ and $(7),(8)$ are satisfied.

In this example, $0 \notin \sigma(S)$ but $\inf \sigma(S)=-\infty$ and $\sup \sigma(S)=\infty$ so only the results in Section 5 of this report are applicable to the problem (7), (8). See [10], [79] . 


\section{Bifurcation from the infimum of the spec- trum}

In this section we consider the equation (2) under the additional hypothesis that

(B) There exist $\lambda_{0} \in \Re$ and $v>0$ such that $A-\lambda_{0} L \geq v I$.

Setting

$$
\Lambda=\inf \{\langle A u, u\rangle /\langle L u, u\rangle: u \in H \text { and } u \neq 0\}
$$

we have that $\Lambda>\lambda_{0}>-\infty$ and

$$
\langle(A-\lambda L) u, u\rangle \geq \alpha(\lambda)\|u\|^{2} \text { for all } u \in H \text { and } \lambda_{0} \leq \lambda<\Lambda
$$

where $\alpha(\lambda)=v(\Lambda-\lambda) /\left(\Lambda-\lambda_{0}\right)$.

In terms of the construction based on the equation (3), the condition (B) amounts to supposing that the operator $S$ is bounded below and then $\Lambda=\inf \sigma(S)$.

We present two ways of approaching the problem of bifurcation from $\Lambda$. In the first method we fix a value of $\lambda<\Lambda$ and then seek non-zero critical points of the functional $F(\lambda, u)=J(u)-(1 / 2) \lambda\langle L u, u\rangle$. Bifurcation is established by considering values of $\lambda$ close to $\Lambda$ and giving conditions under which the norms of these critical points tend to zero as $\lambda$ approaches $\Lambda$. The other method is to look for stationary points of $J$ subject to the constraint $\langle L u, u\rangle=r^{2}$ for fixed $r>0$. In this case the parameter $\lambda$ appears as a Lagrange multiplier and bifurcation is established by showing that as $r$

tends to zero so does the norm of the associated critical point whereas the corresponding values of $\lambda$ tend to $\Lambda$. 


\subsection{The fixed $\lambda$ approach}

We consider the equation (2) under the hypotheses (H1),(H2) and (B). Fixing $\lambda<\Lambda$ we seek non-zero critical points of the functional, $F(\lambda, \cdot): H \longrightarrow \Re$, defined by

$$
F(\lambda, u)=(1 / 2)\langle(A-\lambda L) u, u\rangle-\varphi(u)
$$

Note that since $\lambda<\Lambda$, the expression $\langle(A-\lambda L) u, v\rangle$ defines a scalar product on $H$ which is equivalent to $\langle\cdot, \cdot\rangle$. In general the functional $F(\lambda, \cdot)$ is neither bounded above nor below on $H$ and furthermore $u=0$ is a critical point. Following a well-known path attributed to Nehari, we circumvent these difficulties by considering the restriction of $F(\lambda, \cdot)$ to the set

$$
M(\lambda)=\{u \in H: G(\lambda, u)=0 \text { and } u \neq 0\}
$$

where

$$
G(\lambda, u)=\langle(A-\lambda L) u, u\rangle-\langle N(u), u\rangle
$$

which clearly contains all the non-zero critical points of $F(\lambda, \cdot)$.

On $M(\lambda)$, we have that

$$
F(\lambda, u)=(1 / 2)\langle N(u), u\rangle-\varphi(u)
$$

and this leads us to introduce the following auxiliary functionals,

$$
\Phi(u)=\langle N(u), u\rangle=\varphi^{\prime}(u) u \text { for } u \in H
$$

and

$$
\tilde{\varphi}(u)=(1 / 2)\langle N(u), u\rangle-\varphi(u)=\frac{1}{2} \Phi(u)-\varphi(u) \text { for } u \in H .
$$

Then we set

$$
\begin{aligned}
m(\lambda) & =\inf \{F(\lambda, u): u \in M(\lambda)\} \\
& =\inf \{\tilde{\varphi}(u): u \in M(\lambda)\} .
\end{aligned}
$$

From these definitions it follows easily that, for all $u \in H$,

$$
\begin{aligned}
G(\lambda, u) & =D_{u} F(\lambda, u) u \\
& =2\{F(\lambda, u)-\tilde{\varphi}(u)\}
\end{aligned}
$$


The structure of the set $M(\lambda)$ is regulated by the behaviour of $\varphi$ through the conditions which we now impose.

(G) The functional $\Phi \in C^{1}(H, \Re)$ and the functions $\nabla \varphi$ and $\nabla \Phi$ take bounded subsets of $H$ into bounded subsets of $H$. Furthermore, there exist constants $K>0$ and $q \geq p>2$ such that, for all $u \in H \backslash\{0\}$,

$$
\begin{aligned}
\varphi^{\prime}(u) u & \geq p \varphi(u)>0 \\
\tilde{\varphi}^{\prime}(u) u & \geq p \tilde{\varphi}(u) \\
\text { and } \tilde{\varphi}(u) & \leq K\left\{\|u\|^{p}+\|u\|^{q}\right\} .
\end{aligned}
$$

It follows that $\tilde{\varphi}(u) \geq(p / 2-1) \varphi(u)>0$ for all $u \in H \backslash\{0\}$.

For $u \in M(\lambda)$, we find that

1. $D_{u} G(\lambda, u) u=-2 \tilde{\varphi}^{\prime}(u) u>0$ and consequently $M(\lambda)$ is a $\mathrm{C}^{1}$-manifold.

2. $\alpha(\lambda)\|u\|^{2} \leq\langle(A-\lambda L) u, u\rangle=\varphi^{\prime}(u) u=2\{\varphi(u)+\tilde{\varphi}(u)\}$

$\leq 2 p \tilde{\varphi}(u) /(p-2) \leq 2 p K\left\{\|u\|^{p}+\|u\|^{q}\right\} /(p-2)$ and so there exists $\delta(\lambda)>0$ such that $\|u\| \geq \delta(\lambda)$.

3. $\tilde{\varphi}(u) \geq(p-2) \varphi^{\prime}(u) u / 2 p=(p-2) \alpha(\lambda)\|u\|^{2} / 2 p \geq(p-2) \alpha(\lambda) \delta(\lambda)^{2} / 2 p$ and hence we have that $m(\lambda)>0$.

4. $\|u\|^{2} \leq 2 p F(\lambda, u) /[(p-2) \alpha(\lambda)]$.

The variational principle that we exploit can now be stated as follows.

If $u_{\lambda} \in M(\lambda)$ is such that $F\left(\lambda, u_{\lambda}\right)=m(\lambda)$ then $\left(\lambda, u_{\lambda}\right)$ satisfies equation (2) and $0<\|u\|^{2} \leq 2 p m(\lambda) /[(p-2) \alpha(\lambda)]$.

Indeed, if $u_{\lambda} \in M(\lambda)$ is such that $F\left(\lambda, u_{\lambda}\right)=m(\lambda)$ then there is a Lagrange multiplier $\xi$ such that $D_{u} F\left(\lambda, u_{\lambda}\right)=\xi D_{u} G\left(\lambda, u_{\lambda}\right)$. But then,

$$
\xi D_{u} G\left(\lambda, u_{\lambda}\right) u_{\lambda}=D_{u} F\left(\lambda, u_{\lambda}\right) u_{\lambda}=0
$$

and so $\xi=0$.

In view of this bifurcation for equation (2) can be established by giving conditions under which 
1. $m(\lambda)$ is attained for all $\lambda<\Lambda$,

and

2. $m(\lambda) /(\Lambda-\lambda) \longrightarrow 0$ as $\lambda \longrightarrow \Lambda$.

The first step requires some kind of compactness.

The simplest situation occurs when $\Phi$ is weakly sequentially continuous.

Theorem 4.1 Let the conditions (H1),(H2),(B) and (G) be satisfied and suppose that

(a) $\tilde{\varphi}: H \longrightarrow \Re$ is weakly sequentially lower semicontinuous and that

(b) $\Phi: H \longrightarrow \Re$ is weakly sequentially continuous.

Then for every $\lambda<\Lambda$, there exists an element $u_{\lambda} \in M(\lambda)$ such that $F\left(\lambda, u_{\lambda}\right)=$ $m(\lambda)$. Furthermore, $\left(\lambda, u_{\lambda}\right)$ is a solution of equation (2) and $0<\left\|u_{\lambda}\right\|^{2} \leq$ $2 p m(\lambda) /[(p-2) \alpha(\lambda)]$.

In particular, $\Lambda \in B$ provided that $m(\lambda) /(\Lambda-\lambda) \longrightarrow 0$ as $\lambda \longrightarrow \Lambda$. In this case there is bifurcation to the left and it is of order $\theta$ for any $\theta$ such that $m(\lambda) /(\Lambda-\lambda)^{1+2 \theta} \longrightarrow 0$ as $\lambda \longrightarrow 0$.

See Theorem 3.3 of [75]. Briefly the proof goes as follows.

Consider a minimizing sequence $\left\{u_{n}\right\} \subset M(\lambda)$ such that $\tilde{\varphi}\left(u_{n}\right) \longrightarrow m(\lambda)$. By the point 2, we see that $\left\{u_{n}\right\}$ is bounded in $H$ and so, by passing to a subsequence, we may suppose that $u_{n} \rightarrow u$ weakly in $H$.

From (b) and point 2 we conclude that

$$
\Phi(u)=\lim \Phi\left(u_{n}\right)=\lim \left\langle(A-\lambda L) u_{n}, u_{n}\right\rangle \geq \alpha(\lambda) \delta(\lambda)^{2}>0
$$

and hence that $u \neq 0$.

Since $\langle(A-\lambda L) u, u\rangle$ is a convex function of $u$ for $\lambda<\Lambda$, we also have that

$$
\langle(A-\lambda L) u, u\rangle \leq \liminf \left\langle(A-\lambda L) u_{n}, u_{n}\right\rangle=\liminf \Phi\left(u_{n}\right)=\Phi(u) .
$$

From this we deduce that there is a number $s \in(0,1]$ such that $s u \in$ $M(\lambda)$. But then

$$
0<m(\lambda) \leq \tilde{\varphi}(s u) \leq \tilde{\varphi}(u) \leq \liminf \tilde{\varphi}\left(u_{n}\right)=m(\lambda)
$$


Consequently $s=1$ and the result follows.

The compactness can be influenced by the invariance of the functionals with respect to a group action on $H$. There are two complementary situations which we now describe.

Let $O(H)$ denote the group (with respect to composition) of all isometric isomorphisms of $H$ and consider a subgroup, $G$, of $O(H)$. For $u \in H$, the orbit containing $u$ generated by $G$ is

$$
\Theta(u)=\{T u: T \in G\}
$$

The subspace of all $G$-invariant elements of $H$ is denoted by

$$
H_{G}=\{u \in H: \Theta(u)=\{u\}\} .
$$

Consider a functional $K \in C^{1}(H, \Re)$. Recall that it is said to be $G$-invariant if $K(T u)=K(u)$ for all $u \in H$ and all $T \in G$. It follows that $T^{*}(\nabla K) T=$ $\nabla K$ for all $T \in G$ and so $\|\nabla K(v)\|=\|\nabla K(u)\|$ for all $v \in \Theta(u)$. In particular, $\nabla K: H \longrightarrow H$ is equivariant and $\nabla K(u)=T^{*} \nabla K(u)$ for all $u \in H_{G}$. Thus $\nabla K(u) \in H_{G}$ whenever $u \in H_{G}$.

Given the appropriate invariance we may apply the previous result on the subspace $H_{G}$.

Corollary 4.2 Let the conditions (H1),(H2),(B) and (G) be satisfied and suppose that there is a subgroup, $G$, of $O(H)$ such that $A, L$ and $N$ are $G$-equivariant, that

(a) $\tilde{\varphi}: H_{G} \longrightarrow \Re$ is weakly sequentially lower semicontinuous and that

(b) $\Phi: H_{G} \longrightarrow \Re$ is weakly sequentially continuous.

If $H_{G} \neq\{0\}$, then for every $\lambda<\Lambda$, there exists a solution $\left(\lambda, u_{\lambda}\right)$ of equation (2) where $u_{\lambda} \in H_{G}$ and $0<\left\|u_{\lambda}\right\|^{2} \leq 2 p m_{G}(\lambda) /[(p-2) \alpha(\lambda)]$ where $m_{G}(\lambda)=$ $\inf \left\{F(\lambda, u): u \in M(\lambda) \cap H_{G}\right\}$.

In particular, $\Lambda \in B$ provided that $m_{G}(\lambda) /(\Lambda-\lambda) \longrightarrow 0$ as $\lambda \longrightarrow \Lambda$.

For all $\lambda \in \Re, F(\lambda, \cdot): H \longrightarrow \Re$ is $G$-invariant, as are $\varphi, \tilde{\varphi}$ and $\Phi$. Applying the theorem to the restrictions of these functionals to $H_{G}$, we obtain $u_{\lambda} \in$ $H_{G}$ such that $\nabla f\left(\lambda, u_{\lambda}\right)=0$ and $0<\left\|u_{\lambda}\right\|^{2} \leq 2 p m_{G}(\lambda) /[(p-2) \alpha(\lambda)]$ where $f(\lambda, \cdot)$ is the restriction of $F(\lambda, \cdot)$ to $H_{G}$ and $m_{G}(\lambda)=\inf \left\{f(\lambda, u): u \in M(\lambda) \cap H_{G}\right\}$. It follows that $\nabla F\left(\lambda, u_{\lambda}\right)=0$. 
In Section 6 where $H$ is the usual Sobolev space $H^{1}\left(\Re^{N}\right)$ we show how the group $G$ can be chosen so that $H_{G}$ consists of the radially symmetric elements of $H^{1}\left(\Re^{N}\right)$ and hence the above result can be used to deal with equations involving radial symmerty. For problems involving periodicity there is also an obvious choice of $G$ on $H^{1}\left(\Re^{N}\right)$ but in this case the above proceedure fails since now $H_{G}=\{0\}$.

An alternative way in which invariance can be used even when the space $H_{G}$ is trivial is available provided there is weak orbital compactness in the following sense.

Definition 4.3 For a subgroup $G$ of $O(H)$ we say that $K \in C^{1}(H, \Re)$ is weakly G-compact if

(i) $K$ is $G$-invariant, and

(ii) from every bounded sequence $\left\{u_{n}\right\}$ in $H$ such that $K\left(u_{n}\right) \longrightarrow c \neq K(0)$ and $\left\|\nabla K\left(u_{n}\right)\right\| \longrightarrow 0$ we can extract a subsequence $\left\{u_{n_{i}}\right\}$ and select elements $v_{n} \in \Theta\left(u_{n}\right)$ such that $v_{n_{i}} \rightarrow v$ weakly in $H$ where $v \neq 0$ and $\nabla K(v)=0$.

Theorem 4.4 Let the conditions (H1),(H2),(B) and (G) be satisfied and suppose that there is a subgroup, $G$, of $O(H)$ such that $A, L$ and $N$ are $G$-equivariant and that $F(\lambda, \cdot): H \longrightarrow \Re$ is weakly $G$-compact for all $\lambda \in \Re$.

Then for every $\lambda<\Lambda$, there exists a solution $\left(\lambda, u_{\lambda}\right)$ of equation (2) where $0<\left\|u_{\lambda}\right\|^{2} \leq 2 p m(\lambda) /[(p-2) \alpha(\lambda)]$.

In particular, $\Lambda \in B$ provided that $m(\lambda) /(\Lambda-\lambda) \longrightarrow 0$ as $\lambda \longrightarrow \Lambda$.

Using Ekeland's variational principle, [9], [63], [83], it is shown in Lemma 3.4 of $[75]$ that there is a sequence $\left\{u_{n}\right\} \subset M(\lambda)$ such that

$$
\begin{gathered}
F\left(\lambda, u_{n}\right) \longrightarrow m(\lambda)>0=F(\lambda, 0), \\
\left\|\nabla F\left(\lambda, u_{n}\right)\right\| \longrightarrow 0 \text { and } u_{n} \rightarrow u \text { weakly in } H .
\end{gathered}
$$

The weak $G$-compactness of $F(\lambda, \cdot)$ ensures that, by passing to a subsequence, we may suppose that $u \neq 0$ and that $\nabla F(\lambda, u)=0$. Since $\left\|u_{n}\right\|^{2} \leq p /[(p-$ 2) $\alpha(\lambda)] F\left(\lambda, u_{n}\right)$ the conclusion follows by setting $u_{\lambda}=u$. 
In the absence of any suitable symmetries the necessary compactness can sometimes by established through comparison.

Let $\psi$ be a second functional satisfying the conditions ( $\left.\mathrm{H}_{2}\right)$ and $(\mathrm{G})$. The corresponding auxiliary functionals are denoted by $\Psi$ and $\tilde{\psi}$ respectively and we set

$$
m_{\psi}(\lambda)=\inf \left\{\tilde{\psi}(u): u \in M_{\psi}(\lambda)\right\}
$$

where $M_{\psi}(\lambda)=\left\{u \in H:\langle(A-\lambda L) u, u\rangle=\psi^{\prime}(u) u\right.$ and $\left.u \neq 0\right\}$.

Theorem 4.5 Let the conditions (H1),(H2),(B) and (G) be satisfied and suppose that

(a) $\tilde{\varphi}: H \longrightarrow \Re$ is weakly sequentially lower semicontinuous, and that

(b) $N: H \longrightarrow H$ is weakly sequentially continuous.

Suppose also that there is a functional $\psi$ satisfying (H2) and $(G)$ such that

(c) $\varphi-\psi$ and $\Phi-\Psi: \longrightarrow \Re$ are weakly sequentially continuous at 0 , and

(d) $m(\lambda)<m_{\psi}(\lambda)$ for all $\lambda<\Lambda$.

Then for every $\lambda<\Lambda$, there exists an element $u_{\lambda} \in M(\lambda)$ such that $F\left(\lambda, u_{\lambda}\right)=$ $m(\lambda)$. Furthermore, $\left(\lambda, u_{\lambda}\right)$ is a solution of equation (2) and $0<\left\|u_{\lambda}\right\|^{2} \leq$ $2 p m(\lambda) /[(p-2) \alpha(\lambda)]$.

In particular, $\Lambda \in B$ provided that $m(\lambda) /(\Lambda-\lambda) \longrightarrow 0$ as $\lambda \longrightarrow \Lambda$.

This is Theorem 3.6 of [75]. Note that (b) ensures that $\nabla \varphi: H \longrightarrow H$ is bounded. The proof runs along the following lines.

Using Ekeland's variational principle it is shown in Lemma 3.4 of [75] that there is a sequence $\left\{u_{n}\right\} \subset M(\lambda)$ such that $F\left(\lambda, u_{n}\right) \longrightarrow m(\lambda)>0=F(\lambda, 0)$, $\left\|\nabla F\left(\lambda, u_{n}\right)\right\| \longrightarrow 0$ and $u_{n} \rightarrow u$ weakly in $H$.

It follows easily that $(\lambda, u)$ satisfies the equation (2) and so it suffices to show that $u \neq 0$.

There exists $s_{n}>0$ such that $s_{n} u_{n} \in M_{\psi}(\lambda)$. If $u=0$, we find that

(i) $\lim \left[\tilde{\psi}\left(s_{n} u_{n}\right)-\tilde{\psi}\left(u_{n}\right)\right]=0$ and (ii) $\lim \left[\tilde{\psi}\left(u_{n}\right)-\tilde{\varphi}\left(u_{n}\right)\right]=0$.

But

$$
m_{\psi}(\lambda) \leq \tilde{\psi}\left(s_{n} u_{n}\right)=\left[\tilde{\psi}\left(s_{n} u_{n}\right)-\tilde{\psi}\left(u_{n}\right)\right]+\left[\tilde{\psi}\left(u_{n}\right)-\tilde{\varphi}\left(u_{n}\right)\right]+\tilde{\varphi}\left(u_{n}\right)
$$


and hence

$$
m_{\psi}(\lambda) \leq m(\lambda) \text { if } u=0 .
$$

Since this contradicts (e) we conclude that $u \neq 0$.

In the next subsection we show how group invariance and comparison can be combined to obtain useful criteria for bifurcation. Similar results could easily be formulated in the present context.

In order to obtain strong results concerning nodal solutions of differential equations, Ruppen ([62], [61]) has shown how to establish the bifurcation of many branches of solutions from $\Lambda$. To describe his result we begin by strengthening the assumption $(\mathrm{G})$ and then we introduce some special subsets of $H^{k}$. When $H$ is a space of functions defined on $\Re^{N}$, the elements $\left(u^{1}, \ldots, u^{k}\right)$ of these sets are formed of functions $u^{j}$ in $H$ such that the sets $\left\{x: u^{j}(x) \neq 0\right\}$ are mutually disjoint and so $u^{1}+\ldots+u^{k}$ is a candidate for a solution with at least $k-1$ nodes (in $\Re$ ) or $k$ nodal regions (in $\Re^{N}$ for $N \geq 2$ ).

$\left(\mathrm{G}^{*}\right)$ The condition $(\mathrm{G})$ is satisfied and, for all $u \in H \backslash\{0\}$,

$$
q \tilde{\varphi}(u) \geq \tilde{\varphi}^{\prime}(u) u \geq p \tilde{\varphi}(u) .
$$

Next we set

$$
M^{k}(\lambda)=\left\{\left(u^{1}, \ldots, u^{k}\right): u^{i} \in M(\lambda) \text { for } i=1, \ldots, k\right\}
$$

with the metric induced by

$$
\left\|\left(u^{1}, \ldots, u^{k}\right)\right\|=\sqrt{\left\|u^{1}\right\|^{2}+\ldots+\left\|u^{k}\right\|^{2}} .
$$

Let $i: H^{k} \rightarrow H$ denote the function defined by

$$
i\left(u^{1}, \ldots, u^{k}\right)=u^{1}+\ldots+u^{k} .
$$

Then we consider closed subsets, $\Omega^{k}(\lambda)$, of $M^{k}(\lambda)$ having the following properties for all $\left(u^{1}, \ldots, u^{k}\right) \in \Omega^{k}(\lambda)$. 
1. $\left\|i\left(u^{1}, \ldots, u^{k}\right)\right\|=\left\|\left(u^{1}, \ldots, u^{k}\right)\right\|$.

2. $S\left(i\left(u^{1}, \ldots, u^{k}\right)\right)=S\left(u^{1}\right)+\ldots+S\left(u^{k}\right)$ where $S$ denotes any of the functions $\varphi, \Phi, \tilde{\varphi}, F(\lambda, \cdot), G(\lambda, \cdot)$.

3. $i\left(\Omega^{k}(\lambda)\right) \subset M(\lambda)$.

4. $D_{u} F\left(\lambda, i\left(u^{1}, \ldots, u^{k}\right)\right) u^{j}=0$ for $j=1, \ldots, k$.

5. $\left\langle\nabla \varphi\left(i\left(u^{1}, \ldots, u^{k}\right)\right), u^{j}\right\rangle=\left\langle\nabla \varphi\left(u^{j}\right), u^{j}\right\rangle$ for $j=1, \ldots, k$.

6. $\left\langle i\left(u^{1}, \ldots, u^{k}\right), u^{j}\right\rangle=\left\langle u^{j}, u^{j}\right\rangle$ for $j=1, \ldots, k$.

For such a set $\Omega^{k}(\lambda)$, we define a critical value, $c^{k}(\lambda)$, by

$$
c^{k}(\lambda)=\inf \left\{\tilde{\varphi}\left(i\left(u^{1}, \ldots, u^{k}\right)\right):\left(u^{1}, \ldots, u^{k}\right) \in \Omega^{k}(\lambda)\right\} .
$$

Note that for $\left(u^{1}, \ldots, u^{k}\right) \in \Omega^{k}(\lambda)$, we have that

$$
F\left(\lambda, i\left(u^{1}, \ldots, u^{k}\right)\right)=\tilde{\varphi}\left(i\left(u^{1}, \ldots, u^{k}\right)\right) .
$$

It is easy to see that a minimizing sequence is bounded and so we can assume that there is a sequence $\left\{\left(u^{1}, \ldots, u^{k}\right)_{n}\right\} \subset \Omega^{k}(\lambda)$ such that

$\tilde{\varphi}\left(i\left(u^{1}, \ldots, u^{k}\right)_{n}\right) \rightarrow c^{k}(\lambda), u_{n}^{i} \rightarrow u^{i}$ for $i=1, \ldots, k$ and $i\left(u^{1}, \ldots, u^{k}\right)_{n} \rightarrow$ $u^{1}+\ldots+u^{k}$ weakly in $H$.

We now suppose that it can be chosen so as to have the following additional properties.

$\left(\mathrm{A}_{k}\right)$ There is a sequence $\left\{\left(u^{1}, \ldots, u^{k}\right)_{n}\right\} \subset \Omega^{k}(\lambda)$ such that $\tilde{\varphi}\left(i\left(u^{1}, \ldots, u^{k}\right)_{n}\right) \rightarrow c^{k}(\lambda), u_{n}^{i} \rightarrow u^{i}$ for $i=1, \ldots, k$ and $i\left(u^{1}, \ldots, u^{k}\right)_{n} \rightarrow$ $u^{1}+\ldots+u^{k}$ weakly in $H$. Furthermore, there exists $s \in\{0, \ldots, k\}$ such that $u^{i}=0$ for $i \leq s$ whereas, for $i \geq s+1, \tilde{\varphi}\left(u_{n}^{i}\right) \rightarrow \tilde{\varphi}\left(u^{i}\right)$ and there exist $t^{i} \in(0,1]$ such that $\left(t^{s+1} u^{s+1}, \ldots, t^{k} u^{k}\right) \in \Omega^{(k-s)}(\lambda)$.

Finally we assume that the variational principle yields solutions of (2).

$\left(\mathrm{L}_{k}\right)$ If $\tilde{\varphi}\left(i\left(u^{1}, \ldots, u^{k}\right)\right)=c^{k}(\lambda)$ for some $\left(u^{1}, \ldots, u^{k}\right) \in \Omega^{k}(\lambda)$, then there exists $u_{\lambda}^{k} \in i\left(\Omega^{k}(\lambda)\right)$ such that $\left(\lambda, u_{\lambda}^{k}\right) \in E, \tilde{\varphi}\left(u_{\lambda}^{k}\right)=c^{k}(\lambda)$ and $\left\|u_{\lambda}^{k}\right\|=\left\|\left(u^{1}, \ldots, u^{k}\right)\right\|$.

Ruppen's results can now be stated. 
Theorem 4.6 Let the conditions (H1),(H2),(B) and $\left(G^{*}\right)$ be satisfied and suppose that

(a) $\tilde{\varphi}: H \longrightarrow \Re$ is weakly sequentially lower semicontinuous and that

(b) $\Phi: H \longrightarrow \Re$ is weakly sequentially continuous.

For some $k \geq 2$, suppose also that for, every $\lambda<\Lambda$, the conditions $\left(A_{k}\right)$ and $\left(L_{k}\right)$ hold.

Then for every $\lambda<\Lambda$, thers exists a solution $\left(\lambda, u_{\lambda}^{k}\right)$ of equation (2) where $u_{\lambda}^{k} \in i\left(\Omega^{k}(\lambda)\right)$ and $0<\left\|u_{\lambda}^{k}\right\|^{2} \leq 2 p c^{k}(\lambda) /[(p-2) \alpha(\lambda)]$. If $c^{k}(\lambda) /(\Lambda-\lambda) \rightarrow 0$ as $\lambda \rightarrow \Lambda$, these solutions bifurcate from $\Lambda$.

Non-compact cases can be dealt with by comparison.

Theorem 4.7 Let the conditions (H1),(H2),(B) and $\left(G^{*}\right)$ be satisfied and suppose that

(a) $\tilde{\varphi}: H \longrightarrow \Re$ is weakly sequentially lower semicontinuous and that

(b) $N: H \longrightarrow H$ is weakly sequentially continuous.

Suppose also that there is a functional $\psi$ satisfying (H2) and $\left(G^{*}\right)$ such that

(c) $\varphi-\psi$ and $\Phi-\Psi: \longrightarrow \Re$ are weakly sequentially continuous at 0 .

For some $k \geq 2$, suppose also that for, every $\lambda<\Lambda$, the conditions $\left(A_{k}\right)$ and $\left(L_{k}\right)$ hold with

$$
m(\lambda)<m_{\psi}(\lambda) \text { and } c^{s}(\lambda)<c^{s-1}(\lambda)+m_{\psi}(\lambda) \text { for } s=2, \ldots, k .
$$

Then for every $\lambda<\Lambda$, thers exists a solution $\left(\lambda, u_{\lambda}^{k}\right)$ of equation (2) where $u_{\lambda}^{k} \in i\left(\Omega^{k}(\lambda)\right)$ and $0<\left\|u_{\lambda}^{k}\right\|^{2} \leq 2 p c^{k}(\lambda) /[(p-2) \alpha(\lambda)]$. If $c^{k}(\lambda) /(\Lambda-\lambda) \rightarrow 0$ as $\lambda \rightarrow \Lambda$, these solutions bifurcate from $\Lambda$.

In the statement of the above theorem the subscript $\psi$ signifies that the corresponding quantities are defined using the potential $\psi$ instead of $\varphi$.

\subsection{The fixed norm approach}

As in the preceding section we consider the equation (2) under the hypotheses $(\mathrm{H} 1),(\mathrm{H} 2)$ and (B) but now the value of $\lambda$ is not prescribed in advance. Instead we try to find solutions having a given positive value for $\langle L u, u\rangle$. 
Note that $\langle L u, u\rangle^{1 / 2}$ is a norm on $H$, but $H$ is not necessarily complete with respect to this norm.

For $r>0$ we set

$$
S(r)=\left\{u \in H:\langle L u, u\rangle=r^{2}\right\}
$$

and

$$
\mathcal{M}(r)=\inf \{J(u): u \in S(r)\} .
$$

Clearly if $u \in S(r)$ and $J(u)=\mathcal{M}(r)$, then there exists a Lagrange multiplier $\lambda_{r}$ such that $\nabla J(u)=\lambda_{r} L u$ and so $\left(\lambda_{r}, u\right)$ is a solution of equation (2). Hence we give conditions under which $\mathcal{M}(r)$ is attained and then we estimate $\lambda_{r}$ as $r \longrightarrow 0$. Observe that for $\varphi \equiv 0, \mathcal{M}(r)=\Lambda r^{2} / 2$. The results below deal with the case where $\varphi \neq 0$ and $\mathcal{M}(r)<\Lambda r^{2} / 2$ and they use the following hypotheses.

(K) There exist constants $K>0, n \in \aleph, a_{i} \in[0,1)$ and $b_{i}>1-a_{i}$ for $i=1, . . n$ such that

$$
0 \leq 2 \varphi(u) \leq\langle N(u), u\rangle \leq K \sum_{i=1}^{n}\langle(A-\Lambda L) u, u\rangle^{a_{i}}\langle L u, u\rangle^{b_{i}}
$$

for all $u \in H$.

Setting $\tilde{A}=A-\Lambda L, \tilde{J}(u)=1 / 2\langle\tilde{A} u, u\rangle-\varphi(u)$ and

$$
\widetilde{\mathcal{M}}(r)=\inf \{\tilde{J}(u): u \in S(r)\}=\mathcal{M}(r)-\Lambda r^{2} / 2,
$$

we note that $\tilde{A} \geq 0$ and that $J(u) \leq \Lambda r^{2} / 2 \Leftrightarrow \tilde{J}(u) \leq 0$ for $u \in S(r)$.

Using $(\mathrm{K})$ it is easy to deduce the following estimates.

1. There exists $D>0$ such that $\langle\tilde{A} u, u\rangle \leq D h(\langle L u, u\rangle)$ for all $u$ for which $\tilde{J}(u) \leq 0$ where

$$
h(t)=\sum_{i=1}^{n} t^{b_{i} /\left(1-a_{i}\right)}
$$


2. $v\|u\|^{2} \leq\left\langle\left(A-\lambda_{0} L\right) u, u\right\rangle=\langle\tilde{A} u, u\rangle+\left(\Lambda-\lambda_{0}\right)\langle L u, u\rangle$

$\leq D h(\langle L u, u\rangle)+\left(\Lambda-\lambda_{0}\right)\langle L u, u\rangle$ for all $u$ such that $\tilde{J}(u) \leq 0$, where $v>0$.

3. If $(\lambda, u) \in E, \tilde{J}(u) \leq 0$ and $u \in S(r)$, then

$$
-l(r) \leq \lambda-\Lambda \leq 2 \tilde{J}(u) / r^{2}
$$

where

$$
l(r)=K \sum_{i=1}^{n}\left[D h\left(r^{2}\right)\right]^{a_{i}} r^{2\left(b_{i}-1\right)}
$$

4. For $r>0, \mathcal{M}(r) \leq \Lambda r^{2} / 2, \mathcal{M}(r) / r^{2}$ is a non-increasing function of $r$ and $\mathcal{M}(r) / r^{2} \longrightarrow \Lambda / 2$ as $r \longrightarrow 0$.

Note that $h(t) / t \longrightarrow 0$ as $t \longrightarrow 0$ and hence that $l(r) \longrightarrow 0$ as $r \longrightarrow 0$.

The simplest result in this context can now be given [76], [68], [69].

Theorem 4.8 Let the conditions (H1),(H2),(B) and (K) be satisfied. Suppose that

(a) $\varphi: H \longrightarrow \Re$ is weakly sequentially continuous and

(b) there is an $r_{0} \geq 0$ such that $\mathcal{M}(r)<\Lambda r^{2} / 2$ for all $r>r_{0}$.

Then for each $r>r_{0}$, there exists $u_{r} \in S(r)$ such that $J\left(u_{r}\right)=\mathcal{M}(r)$ and there exists $\lambda_{r}$ such that $\left(\lambda_{r}, u_{r}\right)$ is a solution of equation (2). Furthermore

$$
\begin{gathered}
-l(r) \leq \lambda_{r}-\Lambda \leq 2\left\{\frac{\mathcal{M}(r)}{r^{2}}-\frac{\Lambda}{2}\right\}<0 \\
\text { and }\left\|u_{r}\right\| \leq \sqrt{\left(D h\left(r^{2}\right)+\left(\Lambda-\lambda_{0}\right) r^{2}\right) / v} .
\end{gathered}
$$

In particular, $\Lambda \in B$ provided that $r_{0}=0$. In this case the bifurcation is to the left and is of order $\theta$ for any $\theta$ such that $r /\left[\frac{\mathcal{M}(r)}{r^{2}}-\frac{\Lambda}{2}\right]^{\theta} \longrightarrow 0$ as $r \longrightarrow 0$. 
Fix $r>r_{0}$ and consider a sequence $\left\{u_{n}\right\} \subset S(r)$ such that $J\left(u_{n}\right) \longrightarrow$ $\mathcal{M}(r)$. We may suppose that $\tilde{J}\left(u_{n}\right) \leq 0$ for all $n$ and hence that $\left\{u_{n}\right\}$ is bounded in $H$. Passing to a subsequence we may further suppose that $u_{n} \rightarrow w$ weakly in $H$. By the convexity of $\langle L u, u\rangle$ we have that

$$
\langle L w, w\rangle \leq \liminf \left\langle L u_{n}, u_{n}\right\rangle=r^{2}
$$

and by (a) and the convexity of $\langle\tilde{A} u, u\rangle$, we also have that

$$
\tilde{J}(w) \leq \liminf \tilde{J}\left(u_{n}\right)=\widetilde{\mathcal{M}}(r)<0 .
$$

Hence $w \neq 0$ and $\langle L w, w\rangle>0$.

Setting $t=r / \sqrt{\langle L w, w\rangle}$, we have that $t \geq 1$ and that $t w \in S(r)$. Consequently,

$$
\begin{aligned}
\widetilde{\mathcal{M}}(r) & \leq \tilde{J}(t w) \leq 1 / 2\langle\tilde{A} w, w\rangle t^{2}-\varphi(t w) \\
& \leq t^{2} \tilde{J}(w) \text { by }(\mathrm{K}) \\
& \leq t^{2} \widetilde{\mathcal{M}}(r)
\end{aligned}
$$

and hence $\left(1-t^{2}\right) \widetilde{\mathcal{M}}(r) \leq 0$.

Since $\widetilde{\mathcal{M}}(r)<0$ and $t^{2} \geq 1$ this implies that $t=1$. Thus $w \in S(r)$ and $\tilde{J}(w)=\widetilde{\mathcal{M}}(r)$. Setting $u_{r}=w$, the conclusions follow from the remarks preceding the theorem.

As in the previous section there are variants of this result in the case where the problem is invariant under a group action.

Corollary 4.9 Let the conditions (H1),(H2),(B) and (K) be satisfied and suppose that there is a subgroup, $G$, of $O(H)$ such that $A, L$ and $N$ are $G$ equivariant. Suppose that

(a) $\varphi: H_{G} \longrightarrow \Re$ is weakly sequentially continuous and

(b) there is an $r_{0} \geq 0$ such that $\mathcal{M}_{G}(r)<\Lambda r^{2} / 2$ for all $r>r_{0}$ where $\mathcal{M}_{G}(r)=\inf \left\{J(u): u \in S(r) \cap H_{G}\right\}$.

Then for each $r>r_{0}$, there exists $u_{r} \in S(r) \cap H_{G}$ such that $J\left(u_{r}\right)=$ $\mathcal{M}_{G}(r)$ and there exists $\lambda_{r}$ such that $\left(\lambda_{r}, u_{r}\right)$ is a solution of equation (2). Furthermore

$$
-l(r) \leq \lambda_{r}-\Lambda \leq 2\left\{\frac{\mathcal{M}_{G}(r)}{r^{2}}-\frac{\Lambda}{2}\right\}<0
$$


In particular, $\Lambda \in B$ provided that $r_{0}=0$.

By (b), $H_{G} \neq\{0\}$ and the theorem can be applied to the restriction, $j$, of $J$ to $H_{G}$.

Theorem 4.10 Let the conditions (H1),(H2),(B) and (K) be satisfied and suppose that there is a subgroup, $G$, of $O(H)$ such that $A, L$ and $N$ are $G$ equivariant. Suppose that

(a) $F(\lambda, \cdot): H \longrightarrow \Re$ is weakly $G$-compact for all $\lambda<0$

and

(b) $\mathcal{M}(r)<\Lambda r^{2} / 2$ for all $r>0$.

Then for each $r>0$ there exists a solution, $\left(\lambda_{r}, u_{r}\right)$, of equation (2) such that

$$
\begin{array}{r}
-l(r) \leq \lambda_{r}-\Lambda \leq 2\left\{\frac{\mathcal{M}(r)}{r^{2}}-\frac{\Lambda}{2}\right\}<0 \\
\text { and } 0<\left\|u_{r}\right\| \leq \sqrt{\left(D h\left(r^{2}\right)+\left(\Lambda-\lambda_{0}\right) r^{2}\right) / v}
\end{array}
$$

In particular $\Lambda \in B$.

Fixing $r>0$ and using Ekeland's variational principle we see that there exist $\left\{u_{n}\right\} \subset S(r)$ and $\left\{\lambda_{n}\right\} \subset \Re$ such that $J\left(u_{n}\right) \longrightarrow \mathcal{M}(r)$ and

$$
\left\|\nabla J\left(u_{n}\right)-\lambda_{n} L u_{n}\right\|=\left\|\nabla F\left(\lambda_{n}, u_{n}\right)\right\| \longrightarrow 0 .
$$

Setting $\varepsilon_{n}=\left(A-\lambda_{n} L\right) u_{n}-N\left(u_{n}\right)$, we have that $\left\|\varepsilon_{n}\right\| \longrightarrow 0$ and that

$$
(A-\Lambda L) u_{n}-N\left(u_{n}\right)=\left(\lambda_{n}-\Lambda\right) L u_{n}+\varepsilon_{n}
$$

Hence, $2 \tilde{J}\left(u_{n}\right) \geq\left(\lambda_{n}-\Lambda\right) r^{2}+\left\langle\varepsilon_{n}, u_{n}\right\rangle$ where

$$
v\left\|u_{n}\right\|^{2} \leq D h\left(r^{2}\right)+\left(\Lambda-\lambda_{0}\right) r^{2}
$$

since we may assume that $\tilde{J}\left(u_{n}\right)<0$ for all $n \in \aleph$. This implies that $\lambda_{n}<\Lambda$ for all large $n$.

Furthermore,

$$
\left(\lambda_{n}-\Lambda\right) r^{2} \geq-\left\langle N\left(u_{n}\right), u_{n}\right\rangle-\left\|\varepsilon_{n}\right\| \sqrt{\left(D h\left(r^{2}\right)+\left(\Lambda-\lambda_{0}\right) r^{2}\right) / v}
$$




$$
\geq-l(r) r^{2}-\left\|\varepsilon_{n}\right\| \sqrt{\left(D h\left(r^{2}\right)+\left(\Lambda-\lambda_{0}\right) r^{2}\right) / v}
$$

This shows that $\left\{\lambda_{n}\right\}$ is bounded and, passing to a subsequence, we suppose henceforth that $\lambda_{n} \longrightarrow \lambda$ where

$$
-l(r) \leq \lambda-\Lambda \leq 2 \widetilde{\mathcal{M}}(r) / r^{2}<0 .
$$

Now $\left\|\nabla F\left(\lambda, u_{n}\right)\right\| \leq\left\|\varepsilon_{n}\right\|+\left\|\left(\lambda-\lambda_{n}\right) L u_{n}\right\| \longrightarrow 0$ and $F\left(\lambda, u_{n}\right)=J\left(u_{n}\right)-$ $(\lambda / 2) r^{2} \longrightarrow \mathcal{M}(r)-(\lambda / 2) r^{2}<0=F(\lambda, 0)$. By (a) we may suppose that $u_{n} \rightarrow u$ weakly in $H$ where $u \neq 0$ and $\nabla F(\lambda, u)=0$.

Since $0<\|u\| \leq \liminf \left\|u_{n}\right\| \leq \sqrt{\left(D h\left(r^{2}\right)+\left(\Lambda-\lambda_{0}\right) r^{2}\right) / v}$ and since $h(t) / t \longrightarrow 0$ as $t \longrightarrow 0$ this proves the result.

Finally we show that comparison with a perturbation can again be used to obtain the necessary compactness.

Theorem 4.11 Let the conditions (H1),(H2),(B) and (K) be satisfied and suppose that

(a) $N: H \longrightarrow H$ is weakly sequentially continuous.

Suppose also that there is a functional, $\psi$, satisfying (H2) such that

(b) $\psi-\varphi: H \longrightarrow \Re$ is weakly sequentially lower semicontinuous, and

(c) for all $r>0, \mathcal{M}(r)<\mathcal{M}_{\psi}(r) \leq \Lambda r^{2} / 2$ where

$$
\mathcal{M}_{\psi}(r)=\inf \left\{J_{\psi}(u): u \in S(r)\right\} \text { and } J_{\psi}(u)=1 / 2\langle A u, u\rangle-\psi(u) .
$$

Then for each $r>0$ there exists a solution, $\left(\lambda_{r}, u_{r}\right)$, of equation (2) such that

$$
\begin{array}{r}
-l(r) \leq \lambda_{r}-\Lambda \leq 2\left\{\frac{\mathcal{M}(r)}{r^{2}}-\frac{\Lambda}{2}\right\}<0 \\
\text { and } 0<\left\|u_{r}\right\| \leq \sqrt{\left(D h\left(r^{2}\right)+\left(\Lambda-\lambda_{0}\right) r^{2}\right) / v} .
\end{array}
$$

In particular $\Lambda \in B$. 
Fix $r>0$ and consider the sequence $\left\{\left(\lambda_{n}, u_{n}\right)\right\}$ that was constructed using Ekeland's principle in the preceding proof. We have that $\lambda_{n} \longrightarrow \lambda$ and $u_{n} \rightarrow u$ where $u_{n} \in S(r)$,

$$
\begin{aligned}
-l(r) & \leq \lambda-\Lambda \leq 2 \widetilde{\mathcal{M}}(r) / r^{2}<0 \\
\text { and }\left\|u_{n}\right\| & \leq \sqrt{\left(D h\left(r^{2}\right)+\left(\Lambda-\lambda_{0}\right) r^{2}\right) / v} .
\end{aligned}
$$

Furthermore, for all $v \in H$,

$$
\langle v,(A-\lambda L) u-N(u)\rangle=\lim \left\langle v,\left(A-\lambda_{n} L\right) u_{n}-N\left(u_{n}\right)\right\rangle=0
$$

by (a).

If $u=0$, we have

$$
\begin{gathered}
0=[\psi-\varphi](0) \leq \liminf [\psi-\varphi]\left(u_{n}\right)=\liminf \left[J\left(u_{n}\right)-J_{\psi}\left(u_{n}\right)\right] \\
=\mathcal{M}(r)-\limsup J_{\psi}\left(u_{n}\right) \leq \mathcal{M}(r)-\mathcal{M}_{\psi}(r),
\end{gathered}
$$

contradicting (c). Hence $u \neq 0$ and the proof is complete.

The preceding results can be combined to obtain somewhat more explicit conditions for bifurcation for perturbations of equivariant problems.

Corollary 4.12 Let the conditions (H1),(H2),(B) and (K) be satisfied and suppose that

(a) $N: H \longrightarrow H$ is weakly sequentially continuous.

Let $G$ be a subgroup of $O(H)$ for which $A$ and $L$ are $G$-equivariant.

Suppose also that there is a G-invariant functional, $\psi$, satisfying (H2) and (K) such that

(b) $F_{\psi}(\lambda, \cdot): H \rightarrow \Re$ is weakly $G$-compact for all $\lambda<\Lambda$, where

$$
F_{\psi}(\lambda, u)=\frac{1}{2}\langle(A-\lambda L) u, u\rangle-\psi(u),
$$

(c) $\mathcal{M}_{\psi}(r)<\Lambda r^{2} / 2$ for all $r>0$,

(d) $\psi-\varphi: H \longrightarrow \Re$ is weakly sequentially continuous, and

(e) $\psi(u) \leq \varphi(u)$ for all $u \in H$. 
Then, for each $r>0$, there exists a solution, $\left(\lambda_{r}, u_{r}\right)$, of equation (2) such that

$$
\begin{array}{r}
\quad-l(r) \leq \lambda_{r}-\Lambda<2\left\{\frac{\mathcal{M}_{\psi}(r)}{r^{2}}-\frac{\Lambda}{2}\right\}<0 \\
\text { and } 0<\left\|u_{r}\right\| \leq \sqrt{\left(D h\left(r^{2}\right)+\left(\Lambda-\lambda_{0}\right) r^{2}\right) / v} .
\end{array}
$$

In particular $\Lambda \in B$.

Clearly for all $r>0, \mathcal{M}(r) \leq \mathcal{M}_{\psi}(r)$.

Suppose that for some $r>0, \mathcal{M}(r)=\mathcal{M}_{\psi}(r)$. By the proof of Theorem 13 there exists a sequence $\left\{u_{n}\right\} \subset S(r)$ such that $u_{n} \rightarrow u \neq 0$ weakly in $H$ and $J_{\psi}\left(u_{n}\right) \rightarrow \mathcal{M}_{\psi}(r)$. By (e) we have that $J\left(u_{n}\right) \rightarrow \mathcal{M}(r)$. Now we can use Ekeland's principle to replace $\left\{u_{n}\right\}$ by a sequence $\left\{\tilde{u}_{n}\right\}$ such that $\tilde{u}_{n} \rightarrow u, J\left(u_{n}\right) \rightarrow \mathcal{M}(r)$ and $\left\|\nabla J\left(\tilde{u}_{n}\right)-\lambda_{n} L \tilde{u}_{n}\right\| \rightarrow 0$ where $\lambda_{n} \rightarrow \lambda$ with

$$
\begin{aligned}
& -l(r) \leq \lambda-\Lambda \leq 2\left\{\frac{\mathcal{M}(r)}{r^{2}}-\frac{\Lambda}{2}\right\}<0 \\
& \text { and } 0<\|u\| \leq \sqrt{\left(D h\left(r^{2}\right)+\left(\Lambda-\lambda_{0}\right) r^{2}\right) / v}
\end{aligned}
$$

Using (a) we see that $(\lambda, u) \in E$.

On the other hand, if $\mathcal{M}(r)<\mathcal{M}_{\psi}(r)$ for all $r>0$, it follows from Theorem 14 that there is an element $(\lambda, u) \in E$ such that

$$
\begin{aligned}
& -l(r) \leq \lambda-\Lambda \leq 2\left\{\frac{\mathcal{M}(r)}{r^{2}}-\frac{\Lambda}{2}\right\}<0 \\
& \text { and } 0<\|u\| \leq \sqrt{\left(D h\left(r^{2}\right)+\left(\Lambda-\lambda_{0}\right) r^{2}\right) / v} .
\end{aligned}
$$

Corollary 4.13 Let the conditions (H1),(H2),(B) and (K) be satisfied and suppose that

(a) $N: H \longrightarrow H$ is weakly sequentially continuous. Let $G$ be a subgroup of $O(H)$ for which $A$ and $L$ are $G$-equivariant. Suppose also that there is a $G$-invariant functional, $\psi$, satisfying (H2) and (K) such that

(b) $\psi: H_{G} \rightarrow \Re$ is weakly sequentially continuous, 
(c) for all $r>0, \mathcal{M}_{\psi}(r)=\mathcal{M}_{\psi}^{G}(r)<\Lambda r^{2} / 2$ where

$$
\begin{array}{r}
\mathcal{M}_{\psi}(r)=\inf \left\{J_{\psi}(u): u \in S(r)\right\} \text { and } \\
\mathcal{M}_{\psi}^{G}(r)=\inf \left\{J_{\psi}(u): u \in S(r) \cap H_{G}\right\} \text { with } \\
J_{\psi}(u)=\frac{1}{2}\langle A u, u\rangle-\psi(u),
\end{array}
$$

(d) $\psi-\varphi: H \longrightarrow \Re$ is weakly sequentially continuous, and

(e) $\psi(u) \leq \varphi(u)$ for all $u \in H_{G}$.

Then, for each $r>0$, there exists a solution, $\left(\lambda_{r}, u_{r}\right)$, of equation (2) such that

$$
\begin{array}{r}
-l(r) \leq \lambda_{r}-\Lambda<2\left\{\frac{\mathcal{M}_{\psi}(r)}{r^{2}}-\frac{\Lambda}{2}\right\}<0 \\
\text { and } 0<\left\|u_{r}\right\| \leq \sqrt{\left(D h\left(r^{2}\right)+\left(\Lambda-\lambda_{0}\right) r^{2}\right) / v} .
\end{array}
$$

In particular $\Lambda \in B$.

Clearly $\mathcal{M}(r) \leq \inf \left\{J(u): u \in S(r) \cap H_{G}\right\} \leq \mathcal{M}_{\psi}^{G}(r)=\mathcal{M}_{\psi}(r)$.

If $\mathcal{M}(r)<\mathcal{M}_{\psi}^{G}(r)=\mathcal{M}_{\psi}(r)$, the existence of a solution $\left(\lambda_{r}, u_{r}\right)$, satisfying the desired inequalities follows from Theorem 14.

Henceforth we suppose that $\mathcal{M}(r)=\mathcal{M}_{\psi}^{G}(r)=\mathcal{M}_{\psi}(r)$.

It follows from Corollary 12 that there exists $v_{r} \in S(r) \cap H_{G}$ such that $J_{\psi}\left(v_{r}\right)=\mathcal{M}_{\psi}^{G}(r)$. But then $\mathcal{M}(r) \leq J\left(v_{r}\right) \leq J_{\psi}\left(v_{r}\right)=\mathcal{M}_{\psi}^{G}(r)=\mathcal{M}(r)$. This implies that $\left(\lambda_{r}, u_{r}\right) \in E$ where $u_{r}=v_{r}$ and the required inequalities are satisfied. 


\section{$5 \quad$ Bifurcation into spectral gaps}

The hypothesis (B) of the previous section implies that $\left(-\infty, \lambda_{0}\right] \subset \rho(A, L)$ and in terms of the construction based on the equation (3), this amounts to the assumption that the operator $S$ is bounded below. In the present discussion we impose no such restriction but instead we suppose that $\sigma(A, L) \neq \Re$. We denote by $(a, b)$ a maximal interval in $\rho(A, L)$ and the results in this section give conditions ensuring that $b \in B$ and that there is bifurcation to the left. By replacing $A$ by $A-\mu L$ for $\mu \in(a, b)$ it is sufficient to consider the case where $a<0<b$.

For the analysis we require the functional $\varphi$ to be $C^{2}$ and convex on $H$.

(H3) $\varphi \in C^{2}(H, \Re)$ and $\varphi: H \longrightarrow \Re$ is convex.

From the hypotheses (H2) and (H3) it follows that for all $u, v \in H$,

$$
\varphi(v) \geq \varphi(u)+\langle N(u), v-u\rangle
$$

and

$$
\left\langle N^{\prime}(u) v, v\right\rangle \geq 0
$$

In particular we have that $\varphi(v) \geq 0$ for all $v \in H$.

(S) $0 \notin \sigma(A, L)$ and $P L=L P$ where $P$ is the orthogonal projection associated with the positive part of the spectrum of $A$ which is defined below.

Since $0 \in \rho(A, L)$ implies that $0 \notin \sigma(A)$, the spectral theorem for selfadjoint operators shows that there exist a closed subspace $V$ of $H$ and constants $\alpha, \beta \in(0, \infty)$ such that

1. $A(V) \subset V$,

2. $\langle A u, u\rangle \geq \beta\|u\|^{2}$ for all $u \in V$,

3. $\langle A u, u\rangle \leq-\alpha\|u\|^{2}$ for all $u \in V^{\perp}$. 
In this context the following notation will be useful.

$W=V^{\perp}$.

$P: H \longrightarrow V$ is the orthogonal projection of $H$ on to $V$.

$Q=I-P$.

It follows from (H1) and (S) that

$$
A(V)=V \text { and that } A(W)=W .
$$

The second part of $(\mathrm{S})$ asserts that $V$ also reduces $L$. Note that this is always so when $A$ and $L$ are obtained from the equation (3) by the construction in Section 3

Let $(a, b)$ denote the maximal interval in $\rho(A, L)$ containing 0 . From $(\mathrm{H} 1)$ and $(\mathrm{S})$, it now follows that

$$
a=\sup \{\langle A u, u\rangle /\langle L u, u\rangle: u \in W \text { and } u \neq 0\}
$$

and

$$
b=\inf \{\langle A u, u\rangle /\langle L u, u\rangle: u \in V \text { and } u \neq 0\} .
$$

with $a=-\infty \Leftrightarrow W=\{0\}$ and $b=\infty \Leftrightarrow V=\{0\}$.

The situation discussed in the preceding section corresponds to the case where $a=-\infty$ and so $V=H$. To deal with the general case where $V \neq$ $H$, we use the convexity of $\varphi$ to replace the equation (2) by an equivalent problem for $(\lambda, v) \in(a, \infty) \times V$, via a global version of the Lyapunov-Schmidt reduction. This approach is developed in [79] under the following additional assumptions.

(E)There exist $q \geq p>2$ such that for all $u \in H$

$$
q \varphi(u) \geq \varphi^{\prime}(u) u=\langle N(u), u\rangle \geq p \varphi(u)
$$

and furthermore there exist constants $\varepsilon, C, D$ and $E>0$ such that

$$
\|N(u)\| \leq C+D \varphi(u) \text { for all } u \in H
$$

and

$$
\|N(u)\| \leq E \sqrt{\varphi(u)} \text { for all } u \in H \text { with } \varphi(u)<\varepsilon
$$


The criterion for bifurcation from $b$ is based on a quantitative comparison of the quadratic and superquadratic contributions to the functional $F(b, u)$ given by the following condition which is a slight variant of similar conditions introduced in [41], [26], [29].

(T $\delta$ ) We have that $b<-\infty$ and there is a sequence $\left\{u_{n}\right\} \subset H$ such that $\left\|u_{n}\right\|=1, \varphi\left(u_{n}\right)>0$ for all $n \in \aleph$, and

$$
\lim _{n \rightarrow \infty} \frac{\left\langle(A-b L) u_{n}, u_{n}\right\rangle}{\varphi\left(u_{n}\right)^{\delta}}=\lim _{n \rightarrow \infty} \frac{\left\|(A-b L) u_{n}\right\|^{2}}{\varphi\left(u_{n}\right)^{\delta}}=0 .
$$

We can now state the main result of this section.

Theorem 5.1 Let the conditions (H1) to (H3),(S) and (E) be satisfied and suppose that the condition $T(\delta)$ is fulfilled for some number $\delta \geq 1$. Suppose that either

(a) $N: H \longrightarrow H$ is compact, or

(b) for all $\lambda \in(a, b)$ the functional $F(\lambda, \cdot): H \longrightarrow \Re$ is weakly $G$-compact for a subgroup $G$ of $O(H)$ and $\tilde{\varphi}: H \longrightarrow \Re$ is weakly sequentially lower semicontinuous.

Then $b \in B$. In fact there is bifurcation to the left of order $\theta=\left[1-\frac{1}{\delta}\right] /(q-$ 2).

Remark Under the same hypotheses we deduce that $a$ is a bifurcation point for the equation

$$
A u+N(u)=\lambda L u
$$

It suffices to replace $A$ by $-A$ and $\lambda$ by $-\lambda$.

The proof is given in [79], but here we can only outline the main steps.

By a global Lyapunov-Schmidt reduction there is a unique function $g$ belonging to $C^{1}((a, \infty) \times V, W)$ such that an element $(\lambda, v+w)$ in $(a, \infty) \times$ $[V \oplus W]$ satisfies $(2)$ if and only if $w=g(\lambda, v)$. Then setting

$$
f(\lambda, v)=F(\lambda, v+g(\lambda, v)) \text { for } \lambda>a \text { and } v \in V,
$$


it turns out that $(\lambda, v+g(\lambda, v))$ is a solution of equation (2) if and only if $v$ is a critical point of the functional $f(\lambda, \cdot): V \longrightarrow \Re$.

For $\lambda \in(a, b)$, this functional has a strict local minimum at $v=0$ and the existence of another critical point (yielding a non-trivial solution of (2)) can be established by the mountain pass method for which we set

$$
c(\lambda, v)=\inf _{\gamma \in \Gamma} \max _{t \in[0,1]} f(\lambda, \gamma(t))
$$

where $\Gamma=\{\gamma \in C([0,1], V): \gamma(0)=0$ and $\gamma(1)=v\}$.

Using the sequence $\left\{u_{n}\right\}$ given by the condition (T $\delta$ ), a sequence $\left\{v_{n}\right\}$ in $V$ can be defined by $v_{n}=P u_{n} /\left\|P u_{n}\right\|$, at least for large values of $n$.

Furthermore there exists a sequence $\left\{\lambda_{n}\right\}$ having the following properties

1. $0<\lambda_{n}<b$ and $\lambda_{n} \longrightarrow b$ as $n \longrightarrow \infty$,

2. $f\left(\lambda_{n}, v_{n}\right)<0$ for all $n \geq n_{0}$,

3. $0<c\left(\lambda_{n}, v_{n}\right)$ and $c\left(\lambda_{n}, v_{n}\right) /\left(b-\lambda_{n}\right)^{1+2 \theta} \longrightarrow 0$ as $n \longrightarrow \infty$.

Using (a) or (b) we deduce that for $n \geq n_{0}$ there exists $\left(\lambda_{n}, z_{n}\right) \in E$ such that $0 \leq F\left(\lambda_{n}, z_{n}\right) \leq c\left(\lambda_{n}, v_{n}\right)$.

Finally, bifurcation at $b$ is established by showing that there exists a constant $K>0$ such that

$$
\left\|z_{n}\right\| \leq K \sqrt{c\left(\lambda_{n}, z_{n}\right) /\left(b-\lambda_{n}\right)}
$$

In a similar context Heinz [27] has given criteria for the existence of several branches of solutions bifurcating from $b$. For this the conditions (E) and $(\mathrm{T} \delta)$ are strengthened.

(E*)There exist $q \geq p>2$ such that for all $u \in H$

$$
q \varphi(u) \geq \varphi^{\prime}(u) u=\langle N(u), u\rangle \geq p \varphi(u) .
$$

Furthermore $\varphi$ is even and there is a constant $K$ such that

$$
\|N(u)\| \leq\left\{\langle N(u), u\rangle^{(p-1) / p}+\langle N(u), u\rangle^{(q-1) / q}\right\} .
$$

Next we strengthen the hypothesis $(\mathrm{T} \delta)$.

$(\mathrm{T} \delta)_{m}$ We have that $b<-\infty$ and there exist a real normed vector space $Z$ and a sequence $\left\{L_{n}\right\}$ of linear operators, $L_{n}: Z \rightarrow H$, such that 
1. $\operatorname{dim} Z=m$,

2. $\lim _{n \rightarrow \infty}\left\|L_{n} u\right\|=\|u\|$ for all $u \in Z$,

3. there exists a constant $K$ such that,

$$
\|(A-b L) v\|^{2} \leq K|\langle(A-b L) v, v\rangle| \text { for all } v \in \cup_{n=1}^{\infty} L_{n}(Z)
$$

4. there exists a constant $C$ such that

$$
0<\varphi\left(L_{n} u\right) \leq C \text { for all } u \in K \text { with }\|u\|=1
$$

5.

$$
\frac{\sup \left\{\left|\left\langle(A-b L) L_{n} u, L_{n} u\right\rangle\right|: u \in Z \text { and }\|u\|=1\right\}}{\left[\inf \left\{\varphi\left(L_{n} u\right)\right\}: u \in Z \text { and }\|u\|=1\right]^{\delta}} \rightarrow 0 \text { as } n \rightarrow \infty
$$

Theorem 5.2 Let the conditions (H1) to (H3),(S) and $\left(E^{*}\right)$ be satisfied and suppose that there are numbers $\delta \geq 1$ and $m \geq 2$ such that $(T \delta)_{m}$ is fulfilled. Let $N: H \rightarrow H$ be compact.

Then there exist a sequence $\left\{\lambda_{n}\right\} \subset(a, b)$ and $m$ sequences $\left\{u_{n}^{i}\right\}$ for $i=1, \ldots, m$ such that

$$
\left(\lambda_{n}, \pm u_{n}^{i}\right) \in E \text { for all } n \in \aleph \text { and all } i=1, \ldots, m
$$

where

$$
u_{n}^{i} \neq u_{n}^{j} \text { if } i \neq j, \lambda_{n} \rightarrow b \text { and }\left\|u_{n}^{i}\right\| \rightarrow 0 \text { as } n \rightarrow \infty \text { for } i=1, \ldots, m .
$$

See [27] where Heinz bases his approach on a critical point theorem due to Benci, Capozzi and Fortunato.

For non-compact problems Buffoni and Jeanjean [12] have established an interesting result which can be used to deal with perturbations of equivariant equations.

Whereas Theorems 17 and 18 are in the spirit of the fixed $\lambda$ approach to bifurcation, their approach is based on the following variational principle in 
which we use the orthogonal decomposition $H=V \oplus W$ introduced above restricted to the sphere $S(r)=\left\{u \in H:\langle L u, u\rangle=r^{2}\right\}$ which was introduced for the fixed norm approach to bifurcation.

Setting

$$
\tilde{V}=\{v \in V:\langle A v, v\rangle<(b+1)\langle L v, v\rangle\}
$$

we note that $\tilde{V}$ is an open subset of $V$.

Given $r>0$, set

$$
T(r)=\tilde{V} \cap S(r)
$$

Then for $v \in T(r)$, let

$$
C(v)=\left\{u \in H:\langle L u, u\rangle=r^{2}, P u \in[v] \text { and }\|Q u\| \leq \frac{1}{2} \sqrt{\langle L v, v\rangle /\|L\|}\right\}
$$

where $[v]=\{t v: t>0\}$.

Hence, for $v \in T(r)$,

$$
C(v)=\left\{t v+w: t=\sqrt{1-\langle L w, w\rangle / r^{2}}, w \in W \text { and }\|w\| \leq r /(2 \sqrt{\|L\|})\right\}
$$

Finally, for $r>0$ we set

$$
b(r)=\inf _{v \in T(r)} \sup _{u \in C(v)} J(u) .
$$

The first step in this approach is to show that the supremum is attained. For this we introduce the following hypotheses.

(D) $\varphi: H \rightarrow \Re$ is even and the exist positive constants $C, \sigma$ and $\varepsilon$ and a constant $q>2$ such that

$$
\begin{aligned}
\left\|N^{\prime}(u)\right\| & \leq C\|u\|^{\sigma} \text { and } \\
2 \varphi(u) & \leq\langle N(u), u\rangle \leq q \varphi(u)
\end{aligned}
$$

for all $u \in B(\varepsilon)=\{u \in H:\|u\|<\varepsilon\}$.

Using (H1) to (H3), (S) and (D), it follows that for all $r$ small enough and for all $v \in T(r)$, 
1. $C(v) \subset B(\varepsilon))$,

2. there exists a unique element $G(v) \in C(v)$ such that $J(G(v)) \geq J(u)$ for all $u \in C(v)$,

3. $G \in C^{1}(\tilde{B}(r), H), P G(v) \neq 0$ and there is a constant $K$ such that

$$
\|Q G(v)\| \leq K\|v\|^{1+\sigma} \text { for } v \in \tilde{B}(r) \text { where } \tilde{B}(r)=\{v \in \tilde{V}:\|v\|<r\}
$$

4. $\mathrm{Q}\{[A-q(v) L] G(v)-N(G(v))\}=0$ where

$$
q(v)=\langle A G(v)-N(G(v)), P G(v)\rangle /\langle L P G(v), P G(v)\rangle
$$

5.

$$
b(r)=\inf _{v \in T(r)} J(G(v)
$$

Theorem 5.3 Let the conditions (H1) to (H3), (S), (D) and (T1) be satisfied. Suppose also that

(a) $N: H \rightarrow H$ is weakly sequentially continuous, and that

(b) for some subgroup $G$ of $O(H), A, L$ and $N$ are $G$-equivariant and, for all $\lambda \in(a, b), F(\lambda, \cdot): H \rightarrow \Re$ is weakly $G$-compact .

Then $b \in B$.

Using (T1), it follows as in Lemma 4.4 of [12] that there is a sequence $\left\{r_{n}\right\}$ such that $r_{n}>0, r_{n} \rightarrow 0$ and $b\left(r_{n}\right)<b r_{n}^{2} / 2$.

Set $r=r_{n}$ where $r_{n}$ is sufficiently small and consider a minimizing sequence $\left\{v_{k}\right\} \subset T(r)$ such that $J\left(G\left(v_{k}\right)\right) \rightarrow b(r)$. The first step is to show that for all $k$ beyond a certain level $v_{k} \in X(r)$ where

$$
X(r)=\left\{v \in V:\langle L v, v\rangle=r^{2} \text { and }\langle A v, v\rangle \leq\left(b+\frac{1}{2}\right) r^{2}\right\} .
$$

Clearly $X(r)$ is a closed subset of $H$ and $X(r) \subset T(r)$. Henceforth we consider $J \circ G$ restricted to the complete metric space $X(r)$.

As in the proof of Theorem 2.2 in [12], Ekeland's variational principle furnishes a sequence $\left\{v_{k}\right\} \subset X(r)$ such that 
- $J\left(G\left(v_{k}\right)\right) \rightarrow b(r)$

- $\left\|\left[A-q\left(v_{k}\right) L\right] G\left(v_{k}\right)-N\left(G\left(v_{k}\right)\right)\right\| \rightarrow 0$,

- $G\left(v_{k}\right) \rightarrow y_{r}$ weakly in $H$,

- $q\left(v_{k}\right) \rightarrow \lambda_{r}$ where $\lambda_{r} \leq 2 b(r) / r^{2}<b$,

- there is a positive constant $K$ such that $\liminf \left\|G\left(v_{r}\right)\right\| \leq K r$ and $\lambda_{r} \geq b-K r^{\sigma / 2}$.

In particular,

$$
F\left(\lambda_{r}, G\left(v_{k}\right)\right) \rightarrow b(r)-\frac{1}{2} \lambda_{r} r^{2}<r^{2}\left\{b-\lambda_{r}\right\} / 2<0
$$

and $\left\|\nabla F\left(\lambda_{r}, G\left(v_{k}\right)\right)\right\| \rightarrow 0$.

By (b), we can find $z_{k} \in \Theta\left(G\left(v_{k}\right)\right)$ and $u_{r} \in H$ such that

$z_{k} \rightarrow u_{r}$ weakly in $H, 0<\left\|u_{r}\right\| \leq K r$ and $\nabla F\left(\lambda_{r}, u_{r}\right)=0$.

Theorem 5.4 Let the conditions (H1) to (H3), (S), (D) and (T1) be satisfied. Suppose also that

(a) $N: H \rightarrow H$ is weakly sequentially continuous. and that

(b) $A$ and $L$ are $G$-equivariant for some subgroup $G$ of $O(H)$.

Suppose that there is a G-invariant functional $\psi$ satisfying (H3), (D) and (a) such that

(c) $\psi-\varphi: H \rightarrow \Re$ is weakly sequentially continuous at 0 ,

(d) $\psi(u) \leq \varphi(u)$ for all $u \in H$.

Then $b \in B$.

Let

$$
b_{\psi}(r)=\inf _{v \in T(r)} \sup _{u \in C(v)} J_{\psi}(u)
$$

where $J_{\psi}(u)=\frac{1}{2}\langle A u, u\rangle-\psi(u)=J(u)+[\varphi-\psi](u) \geq J(u)$ for all $u \in H$ by (d). Hence $b_{\psi}(r) \geq b(r)$. 
Let $G_{\psi}: T(r) \rightarrow H$ denote the unique function such that

$$
G_{\psi}(v) \in C(v) \text { and } J_{\psi}\left(G_{\psi}(v)\right)=\sup _{u \in C(v)} J_{\psi}(u)
$$

There are two cases to be treated, $b_{\psi}(r)>b(r)$ and $b_{\psi}(r)=b(r)$.

Suppose first that $b_{\psi}(r)>b(r)$. Consider the sequences $\left\{r_{n}\right\}$ and $\left\{v_{k}\right\}$ that are defined in the proof of the preceding theorem. For $r \in\left\{r_{n}\right\}$, it follows from (a) that $\left(\lambda_{r}, y_{r}\right)$ is a solution of equation (2) with $b-K r^{\sigma / 2} \leq$ $\lambda_{r} \leq 2 b(r) / r^{2}<b$ and $\left\|y_{r}\right\| \leq K r$ for a constant $K$ which is independent of $r$. Hence it is sufficient to show that $y_{r} \neq 0$. If $y_{r}=0$, we see from the definition of $C(v)$ that this implies that $v_{k} \rightarrow 0$ in $H$. By Lemma 4.3 of [12] we then have that $G_{\psi}\left(v_{k}\right) \rightarrow 0$ weakly in $H$. But,

$$
\begin{aligned}
b_{\psi}(r) & =\inf _{v \in T(r)} J_{\psi}\left(G_{\psi}(v)\right) \leq J_{\psi}\left(G_{\psi}\left(v_{k}\right)\right) \\
& =J\left(G_{\psi}\left(v_{k}\right)\right)+[\varphi-\psi]\left(G_{\psi}\left(v_{k}\right)\right) \\
& \leq \sup _{u \in C\left(v_{k}\right)} J(u)+[\varphi-\psi]\left(G_{\psi}\left(v_{k}\right)\right)=J\left(G\left(v_{k}\right)\right)+[\varphi-\psi]\left(G_{\psi}\left(v_{k}\right)\right)
\end{aligned}
$$

Letting $k \rightarrow \infty$ and using (c), we conclude that $b_{\psi}(r) \leq b(r)$ contradicting our assumption. Hence $y_{r} \neq 0$.

Next we treat the case where $b_{\psi}(r)=b(r)$. By the proof of the preceding theorem there is a sequence $\left\{v_{k}\right\} \subset X(r)$ such that $J_{\psi}\left(G_{\psi}\left(v_{k}\right)\right) \rightarrow b_{\psi}(r)$ and there are $z_{k} \in \Theta\left(G_{\psi}\left(v_{k}\right)\right)$ and $u_{r} \in H \backslash\{0\}$ such that $z_{k} \rightarrow u_{r}$ weakly in $H$. Let $z_{k}=U_{k} G_{\psi}\left(v_{k}\right)$ where $U_{k} \in O(H)$.

By the $G$-equivariance of $A$ and $L$ it follows from Theorem 2 in Section 7.71 of [47] that for all $U \in G \subset O(H), P U=U P, U T(r)=T(r)$ and $U C(v)=C(U v)$ for all $v \in T(r)$. Thus

$$
\begin{aligned}
J_{\psi}\left(G_{\psi}(U v)\right) & =\sup _{u \in C(U v)} J_{\psi}(u)=\sup _{x \in C(v)} J_{\psi}(U x)=\sup _{x \in C(v)} J_{\psi}(x)=J_{\psi}\left(G_{\psi}(v)\right) \\
& =J_{\psi}\left(U G_{\psi}(v)\right)
\end{aligned}
$$

and consequently, $G_{\psi}(U v)=U G_{\psi}(v)$ for all $v \in T(r)$.

Setting $x_{k}=U_{k} v_{k}$ we see that $x_{k} \in X(r), J_{\psi}\left(G_{\psi}\left(x_{k}\right)\right)=J_{\psi}\left(G_{\psi}\left(v_{k}\right)\right) \rightarrow$ $b_{\psi}(r)$ and $G_{\psi}\left(x_{k}\right)=z_{k}$.

As above we can conclude that $x_{k}-x \neq 0$ since $u_{r} \neq 0$.

Furthermore $J_{\psi}\left(G_{\psi}\left(x_{k}\right)\right) \geq J_{\psi}\left(G\left(x_{k}\right)\right) \geq J\left(G\left(x_{k}\right)\right) \geq b(r)=b_{\psi}(r)$ so $J\left(G\left(x_{k}\right)\right) \rightarrow b(r)$.

Now Ekeland's principle can be employed once again to replace $\left\{x_{k}\right\}$ by a sequence $\left\{\tilde{x}_{k}\right\}$ such that 
- $J\left(G\left(\tilde{x}_{k}\right)\right) \rightarrow b(r)$

- $\tilde{x}_{k} \rightarrow x \neq 0$

- $\left\|\left[A-q\left(\tilde{x}_{k}\right) L\right] G\left(\tilde{x}_{k}\right)-N\left(G\left(\tilde{x}_{k}\right)\right)\right\| \rightarrow 0$,

- $G\left(\tilde{x}_{k}\right) \rightarrow \tilde{y}_{r}$ weakly in $H$,

- $q\left(\tilde{x}_{k}\right) \rightarrow \lambda_{r}$ where $\lambda_{r} \leq 2 b(r) / r^{2}<b$,

- there is a positive constant $K$ such that $\liminf \left\|G\left(\tilde{x}_{r}\right)\right\| \leq K r$ and $\lambda_{r} \geq b-K r^{\sigma / 2}$.

Since $x \neq 0$ we have that $\tilde{y}_{r} \neq 0$ and then, using (a), it follows that $\left(\lambda_{r}, \tilde{y}_{r}\right) \in$ E.

Using the same variational principle Jeanjean has established the existence of infinitely many distinct branches which all bifurcate to the left at $b$.

Theorem 5.5 Let the conditions (H1) to (H3), (S) and (D) be satisfied and suppose that the condition $(T 1)_{m}$ is fulfilled for all $m \in \aleph$. Let $N: H \rightarrow H$ be completely continuous.

Then there exists $r_{0}>0$ such that, for every $r \in\left(0, r_{0}\right)$, there is a sequence $\left\{\left(\lambda_{r}^{i}, u_{r}^{i}\right)\right\}_{i=1}^{\infty}$ of distinct solutions of equation (2) such that $u_{r}^{i} \in S(r)$ for all $i \in \aleph$. Furthermore, there is a constant $K>0$ such that

$$
b-K r^{\sigma / 2}<\lambda_{r}^{i}<b \text { and }\left\|u_{r}^{i}\right\| \leq K r \text { for all } i \in \aleph
$$

See [31]. 


\section{Semilinear elliptic equations}

As mentioned in Section 1 the study of bound states for nonlinear perturbations of Schrödinger operators motivated the formulation of many of the general results on bifurcation from the essential spectrum. Here we summarize most of what is known about this problem. We restrict our attention to the simplest type of nonlinearity, but as can be seen by consulting the references, more general forms can be treated similarly.

Let $\mathcal{H}=L^{2}\left(\Re^{N}\right)$ with the usual norm and, more generally, let $|\cdot|_{q}$ denote the usual norm on $L^{q}\left(\Re^{N}\right)$. We consider square-integrable solutions of the following elliptic equation

$$
-\Delta u(x)+V(x) u(x)-r(x)|u(x)|^{p-2} u(x)=\lambda u(x) \text { for } x \in \Re^{N}
$$

where

and

(A1) $V \in L^{\infty}\left(\Re^{N}\right)$

(A2) $r \in L^{\infty}\left(\Re^{N}\right)$ with $r \geq 0$ and $2<p<2^{*}$ where $2^{*}=\infty$ for $N=1$ and 2 and $2^{*}=2 N /(N-2)$ for $N \geq 3$.

Under the assumption (A1) it is well-known that a self-adjoint operator $S: D(S) \subset L^{2}\left(\Re^{N}\right) \longrightarrow L^{2}\left(\Re^{N}\right)$ is defined by

$$
S u(x)=-\Delta u(x)+V(x) u(x) \text { with } D(S)=H^{2}\left(\Re^{N}\right) .
$$

Furthermore $S$ is bounded below and the form space $(H,\langle\cdot, \cdot\rangle)$ of $\mathrm{S}$ coincides, up to equivalence of norms, with the Sobolev space $H^{1}\left(\Re^{N}\right)$.

Thus, under the hypotheses (A1) and (A2), the equation (10) can be cast in the form (2) where the conditions (H1) and (H2) are satisfied and solutions

of (2) correspond to weak solutions of (10) in the usual sense. Furthermore, solutions of (2) are in fact strong solutions of (10). 
For any $\lambda<\inf V(x)$, we have that

$$
\begin{aligned}
\langle(A-\lambda L) u, u\rangle & =\int\left[|\nabla u|^{2}+(V-\lambda) u^{2}\right] d x \\
& \geq v \int\left[|\nabla u|^{2}+u^{2}\right] d x=v\|u\|^{2}
\end{aligned}
$$

for all $u \in H$ where $v=\min \{1, \inf V-\lambda\}$. When no domain of integration is indicated, it is understood that the integral is taken over $\Re^{N}$.

This shows that the condition (B) is satisfied and that $\Lambda \geq \inf V$.

By the construction in Section 3, we may assume without loss of generality that the condition (S) is satisfied.

On the other hand, for any $u \in H$,

$$
\langle A u, u\rangle \leq \int\left[|\nabla u|^{2}+(\sup V) u^{2}\right] d x=\int|\nabla u|^{2} d x+\sup V\langle L u, u\rangle
$$

and so

$$
\Lambda \leq \sup V+\inf \left\{\frac{\int|\nabla u|^{2} d x}{\int u^{2} d x}: u \in H \backslash\{0\}\right\}=\sup V .
$$

Hence we have that

$$
\inf V \leq \Lambda \leq \sup V .
$$

In order to focus on bifurcation from points in the spectrum of the linear Schrödinger operator $S=-\Delta+V$ which are not eigenvalues, it is convenient to distinguish two broad classes of potential $V$.

(A3) $|x|^{2} V(x) \in L^{\infty}\left(\Re^{N}\right)$ and $V_{-}=0$ for $N=1,2$ whereas $\left|V_{-}\right|_{N / 2}<$ $\frac{N-2}{2(N-1)}$ for $N \geq 3$ where $V_{-}=\max \{0,-V(x)\}$.

(A4) $V(x+k)=V(x)$ for all $x \in \Re^{N}$ and $k \in \mathrm{Z}^{N}$.

We have already shown in example 3 in Section 2 that (A1) and (A4) imply that $\sigma(S)=\sigma_{e}(S)$. In this case $\sigma(S)$ is a union of closed intervals.

The conditions (A1) and (A3) imply that $\sigma_{e}(S)=[0, \infty)$. (See [34], [54].) Furthermore they imply that for all $u \in H$, 


$$
\langle A u, u\rangle \geq \kappa|\nabla u|_{2}^{2} \text { where } \kappa>0
$$

and so $\Lambda=0$ and that $\sigma(S)=[0, \infty)$. This is obvious for $N=1,2$ and for $N \geq 3$ we have that

$$
\begin{aligned}
\langle A u, u\rangle & \geq \int|\nabla u|^{2}+V u^{2} d x \geq \int|\nabla u|^{2}-V_{-} u^{2} d x \\
& \geq|\nabla u|_{2}^{2}-\left|V_{-}\right|_{N / 2}|u|_{2 N /(N-2)}^{2} \\
& \geq|\nabla u|_{2}^{2}-\left|V_{-}\right|_{N / 2} C(N)|\nabla u|_{2}^{2} \geq \kappa|\nabla u|_{2}^{2}
\end{aligned}
$$

where $\kappa=1-\left|V_{-}\right|_{N / 2} C(N)>0$ since we may set $C(N)=2(N-1) /(N-2)$ for $N \geq 3$. (See [8].)

Setting

$$
\varphi(u)=(1 / p) \int_{\Re^{N}} r(x)|u(x)|^{p} d x,
$$

the Sobolev embedding theorems imply that $\varphi \in C^{2}\left(H^{1}\left(\Re^{N}\right), \Re\right)$ and that $R(u):=r|u|^{p-2} u \in L^{2}\left(\Re^{N}\right)$ for all $u \in H^{2}\left(\Re^{N}\right)$. Since

$$
\varphi^{\prime}(u) v=\int_{\Re^{N}} r(x)|u(x)|^{p-2} u(x) v(x) d x \text { for all } u, v \in H^{1}\left(\Re^{N}\right)
$$

it follows that $N(u)=\tilde{L} R(u)$ for all $u \in H^{2}\left(\Re^{N}\right)$.

Furthermore, for all $u, v, w \in H^{1}\left(\Re^{N}\right)$,

$$
\left\langle N^{\prime}(u) v, w\right\rangle=(p-1) \int_{\Re^{N}} r(x)|u(x)|^{p-2} v(x) w(x) d x .
$$

Since $2<p<2^{*}$, we have that

$$
0 \leq p \varphi(u)=\varphi^{\prime}(u) u=\langle N(u), u\rangle \leq|r|_{\infty}|u|_{p}^{p} \leq C|r|_{\infty}\|u\|^{p}
$$

for all $u \in H$. 
Similarly, for $u, v, w \in H$,

$$
\begin{aligned}
\left|\left\langle N^{\prime}(u) v, w\right\rangle\right| & \leq(p-1)|r|_{\infty}|u|_{p}^{(p-2)}|v w|_{p / 2} \\
& \leq(p-1)|r|_{\infty}|u|_{p}^{(p-2)}|v|_{p}|w|_{p} \\
& \leq(p-1) C|r|_{\infty}\|u\|^{(p-2)}\|v\|\|w\|
\end{aligned}
$$

and so

$$
\left\|N^{\prime}(u)\right\| \leq(p-1) C|r|_{\infty}\|u\|^{(p-2)} .
$$

Furthermore the convexity of the function $s \longmapsto|s|^{p}$ on $\Re$ implies that of $\varphi$ on $H$.

Hence we see that the conditions (A1) and (A2) ensure that the hypotheses (H3) and (H4) are satisfied.

As is shown in Lemma 9.1 of [79] the conditions (A1) and (A2) also imply that

(a) $N: H \rightarrow H$ is weakly sequentially continuous and that

(b) there is a constant $K$ such that

$$
\|N(u)\| \leq K \varphi(u)^{1 / p^{\prime}} \text { for all } u \in H
$$

where $\frac{1}{p}+\frac{1}{p^{\prime}}=1$.

Noting that $\frac{1}{2}<\frac{1}{p^{\prime}}<1$, we see that the conditions (A1) and (A2) guarantee that the assumptions (E), (E*) and (D) of Section 5 are all valid in the present context with $\sigma=p-2$.

In the notation of Section 4, we have that

$$
\Phi(u)=p \varphi(u) \text { and } \tilde{\varphi}(u)=\left(\frac{p}{2}-1\right) \varphi(u)
$$

for all $u \in H$.

The convexity of $\varphi$ ensures that $\varphi, \Phi$ and $\tilde{\varphi}$ are all weakly sequentially lower semicontinuous on $H$.

It follows also that the conditions $(\mathrm{G})$ and $\left(\mathrm{G}^{*}\right)$ of Section 4 are fulfilled provided that in addition to (A1) and (A2) we suppose that

$$
\left\{x \in \Re^{N}: r(x)=0\right\} \text { has measure zero. }
$$


The condition (K) of Section 4 is satisfied provided that (A1) to (A3) hold and that $p$ is not too big. In fact by the multiplicative Sobolev inequality of Gagliardo, Nirenberg and Golovkin [76], we have that, for all $u \in H$,

$$
|u|_{q} \leq K(q, N)|\nabla u|_{2}^{\theta}|u|_{2}^{1-\theta}
$$

where $2 \leq q<2^{*}$ and $\theta=N\left(\frac{1}{2}-\frac{1}{q}\right)$. Using the condition (A2), it follows from this that

$$
0 \leq\langle N(u), u\rangle \leq|r|_{\infty}|u|_{p}^{p} \leq K(p, N)^{p}|r|_{\infty}|\nabla u|_{2}^{\beta}|u|_{2}^{p-\beta}
$$

where $\beta=N(p-2) / 2$. From the assumptions (A1) to (A3), we now deduce that, for all $u \in H$,

$$
0 \leq\langle N(u), u\rangle \leq \frac{K(p, N)^{p}|r|_{\infty}}{\kappa^{\beta / 2}}\langle A u, u\rangle^{a}\langle L u, u\rangle^{b}
$$

where $a=N(p-2) / 4$ and $b=(p-2 a) / 2$. This shows that the condition $(\mathrm{K})$ is satisfied provided that (A1) to (A3) hold and that $p<2+\frac{4}{N}$.

Furthermore in the notation of Section 4 we can set $l(r)=K r^{\gamma}$ where $\gamma=1 /\left\{\frac{1}{p-2}-\frac{N}{4}\right\}$.

As far as symmetries are concerned, two cases have been studied extensively, namely, translational and rotational invariance. To discuss these cases we introduce the following notation.

$$
G_{T}=\left\{T_{k}: k \in Z^{N}\right\} \text { where } T_{k} u(x)=u(x-k)
$$

and

$$
G_{R}=\left\{T_{R}: R \in S O(N)\right\} \text { where } T_{R} u(x)=u(R x) .
$$

Clearly $G_{T}$ and $G_{R}$ are the subgroups of $O(H)$ associated with periodicity on a square lattice and radial symmetry respectively. Using the general notation we have that

$$
H_{G_{T}}=\{0\} \text { and } H_{G_{R}}=\{u \in H: u(x)=u(y) \text { whenever }|x|=|y|\} .
$$

The conditions (A1) and (A4) imply that the operators $A$ and $L$ are $G_{T^{-}}$ equivariant. To obtain the $G_{R^{-}}$equivariance of these operators we must replace (A4) by the following property. 
(A5) $V(x)=V(y)$ whenever $|x|=|y|$.

Similarly the equivariance of the operator $N$ is determined by the corresponding symmetry of the coefficient $r$ on $\Re^{N}$.

Let us now turn to the compactness properties of the nonlinearity in this problem. The simplest situation is that in which the coefficient decays to zero at infinity.

(A6) ess $\sup \{|r(x)|:|x| \geq n\} \rightarrow 0$ as $n \rightarrow \infty$.

When the conditions (A2)and (A6) are satisfied it is easy to deduce from the compactness of the Sobolev embeddings on bounded domains that

(i) $\varphi: H \rightarrow \Re$ is weakly sequentially continuous, and

(ii) $N: H \rightarrow H$ is compact.

On the other hand, since there exists a constant $K(N)$ such that

$$
|u(x)| \leq K(N)\|u\||x|^{(1-N) / 2} \text { for all } u \in H_{G_{R}},
$$

similar arguments show that

$$
\varphi: H_{G_{R}} \rightarrow \Re \text { is weakly sequentially continuous }
$$

provided that (A2) is satisfied.

Supposing that the conditions (A1), (A2) and (A4) are satisfied and that

$$
\text { (A7) } r(x+k)=r(x) \text { for all } x \in \Re^{N} \text { and } k \in \mathrm{Z}^{N} \text {, but } r \not \equiv 0 \text {, }
$$

it is shown in Lemma 9.2 of [79] that the functional $F(\lambda, \cdot): H \rightarrow \Re$, defined by

$$
\begin{aligned}
F(\lambda, u) & =\frac{1}{2}\langle(A-\lambda L) u, u\rangle-\varphi(u) \\
& =\frac{1}{2} \int\left\{|\nabla u|^{2}+(V-\lambda) u^{2}\right\} d x-\frac{1}{p} \int r|u|^{p} d x
\end{aligned}
$$


is weakly $G_{T^{-}}$compact for all $\lambda \in \Re$.

The remaining aspect of the general results concerns the estimation the relevant critical values. For the quantities $m(\lambda)$ and $\mathcal{M}(r)$ used in Section 4 respectively this can be done through elementary calculations with test-functions of exponential type. In the results in Section 5 the necessary estimates for $c(\lambda, v)$ are already implied by the condition $\mathrm{T}(\delta)$ so it is sufficient to find test-functions satisfying this latter condition for some value of $\delta \geq 1$. Let us begin with the estimates for $m(\lambda)$.

For $\alpha>0$ and $x \in \Re^{N}$, let

$$
u_{\alpha}(x)=v(\alpha x) \text { where } v(y)=|y| e^{-|y|} .
$$

Clearly $u_{\alpha} \in H$ for all $\alpha>0$, with

$$
\left|u_{\alpha}\right|_{2}^{2}=\alpha^{-N} I_{1} \text { and }\left|\nabla u_{\alpha}\right|_{2}^{2}=\alpha^{2-N} I_{2}
$$

where

$$
I_{1}=|v|_{2}^{2} \text { and } I_{2}=|\nabla v|_{2}^{2} .
$$

Assuming that (A3) is satisfied we see that there is a constant $K>0$ such that

$$
\int V(x) u_{\alpha}(x)^{2} d x \leq K \int|x|^{-2} u_{\alpha}(x)^{2} d x=\alpha^{2-N} I_{3}
$$

where $I_{3}=K \int|y|^{-2} v(y)^{2} d y<\infty$.

On the other hand we obtain a lower bound for $\varphi\left(u_{\alpha}\right)$ by ensuring that the coefficient $r$ does not decay too quickly as $|x| \rightarrow \infty$. The following condition has been used to do this.

$\mathrm{D}(\tau)$ For $\tau \geq 0$, we say that the coefficient $r$ satisfies the condition $\mathrm{D}(\tau)$ provided that there exist a constant $K>0$ and a point $z \in \Re^{N}$ such that $r(x) \geq K|x|^{-\tau}$ for all $x \in C$ where $C=\{t x: t \geq 1$ and $|x-z| \leq 1\}$.

If $r$ satisfies the conditions (A2) and $\mathrm{D}(\tau)$,

$$
\begin{aligned}
p \varphi\left(u_{\alpha}\right) & =\int r(x)\left|u_{\alpha}(x)\right|^{p} d x \geq K \int_{C}|x|^{-\tau}|v(\alpha x)|^{p} d x \\
& =K \alpha^{\tau-N} \int_{\mathcal{C}(\alpha)}|y|^{-\tau}|v(y)|^{p} d y
\end{aligned}
$$


where $\mathcal{C}(\alpha)=\{y: y / \alpha \in C\}=\alpha C \supset C$ for all $\alpha \in(0,1]$.

Hence for $0<\alpha \leq 1$,

$$
\varphi\left(u_{\alpha}\right) \geq \alpha^{\tau-N} I_{4}
$$

where $I_{4}=K \int_{C}|y|^{-\tau}|v(y)|^{p} d y / p$.

To estimate $m(\lambda)$ we suppose that

- (A1) to (A3) are satisfied, that

- $\left\{x \in \Re^{N}: r(x)=0\right\}$ has measure zero,and that

- the condition $\mathrm{D}(\tau)$ is satisfied for some $\tau \in[0,2)$.

Then $\Lambda=0$ and we consider $\lambda<0$. Since $\varphi(u) \neq 0$ for all $u \in H \backslash$ $\{0\}, t u \in M(\lambda)$ for $t>0 \Leftrightarrow t=\left\{\frac{\langle(A-\lambda L) u, u\rangle}{p \varphi(u)}\right\}^{1 /(p-2)}$. Hence

$$
\begin{gathered}
m(\lambda)=\inf \{\tilde{\varphi}(u): u \in M(\lambda)\} \\
=\inf \left\{\left(\frac{p-2}{2}\right)\left\{\frac{\langle(A-\lambda L) u, u\rangle}{p \varphi(u)}\right\}^{p /(p-2)} \varphi(u): u \in H \backslash\{0\}\right\} \\
=K(p) \inf \left\{\frac{\langle(A-\lambda L) u, u\rangle^{p /(p-2)}}{\varphi(u)^{2 /(p-2)}}: u \in H \backslash\{0\}\right\} \\
\leq K(p) \frac{\left\langle\left(A+\alpha^{2} L\right) u_{\alpha}, u_{\alpha}\right\rangle^{p /(p-2)}}{\varphi\left(u_{\alpha}\right)^{2 /(p-2)}} \text { where } \alpha=\sqrt{-\lambda} \\
\leq K(p) \frac{\left\{\left(I_{1}+I_{2}+I_{3}\right) \alpha^{2-N}\right\}^{p /(p-2)}}{\left\{I_{4} \alpha^{\tau-N}\right\}^{2 /(p-2)}} \text { provided that }-1 \leq \lambda<0 .
\end{gathered}
$$

Thus for $-1 \leq \lambda<0, m(\lambda) \leq C|\lambda|^{\rho}$ where $\rho=\left\{\frac{p(2-N)}{2}+N-\tau\right\} /(p-2)$ and consequently, if $p<2+\frac{2(2-\tau)}{N}$,

(i) $m(\lambda) /|\lambda| \rightarrow 0$, and

(ii) for all $\theta<\frac{2(2-\tau)-N(p-2)}{4(p-2)}$, we have that $\frac{m(\lambda)}{|\lambda|^{1+2 \theta}} \rightarrow 0$ as $r \rightarrow 0$. 
Next we estimate the quantity $\mathcal{M}(r)$ which was introduced in the second part of Section 4 using only the conditions (A1) to (A3) and $\mathrm{D}(\tau)$.

For $r>0, t u_{\alpha} \in S(r)$ if $t=r / \sqrt{\alpha^{-N} I_{1}}$ and so

$$
\begin{aligned}
\mathcal{M}(r) & =\inf \left\{\frac{1}{2}\langle A u, u\rangle-\varphi(u): u \in S(r)\right\} \\
& \leq r^{2}\left\langle A u_{\alpha}, u_{\alpha}\right\rangle \alpha^{N} / 2 I_{1}-r^{p} \alpha^{N p / 2} \varphi\left(u_{\alpha}\right) / I_{1}^{p / 2} \\
& \leq r^{2} \alpha^{2}\left(I_{2}+I_{3}\right) / 2 I_{1}-r^{p} \alpha^{N p / 2+\tau-N} I_{4} / I_{1}^{p / 2} \text { for all } \alpha \in(0,1] .
\end{aligned}
$$

Choosing $\alpha=1$ we see that there exists $r_{0} \geq 0$ such that $\mathcal{M}(r) / r^{2}<0$ for all $r>r_{0}$.

However if $p<2+\frac{2(2-\tau)}{N}$, then $\frac{N p}{2}+\tau-N<2$ and so,

(i) for every $r>0$, we can find $\alpha \in(0,1]$ such that $\mathcal{M}(r) / r^{2} \leq 0$,

(ii) for all $\theta<\frac{2(2-\tau)-N(p-2)}{4(p-2)}$, we have that $\frac{r}{\left(\frac{\mathcal{M}(r)}{r^{2}}\right)^{\theta}} \rightarrow 0$ as $r \rightarrow 0$.

Finally we come to the condition $\mathrm{T}(\delta)$ which we shall discuss under the hypothesis that the potential $V$ has the properties (A1) and (A4). Then the spectrum of the Schrödinger operator $-\Delta+V$ consists precisely of those $\lambda \in \Re$ for which the differential equation $-\Delta u+V u=\lambda u$ admits a solution in the form of a Bloch wave, [20]. Let $b \in \sigma(S)$. Then we deduce that there is a non-trivial solution $\xi \in H_{l o c}^{2}\left(\Re^{N}\right) \cap C^{1}\left(\Re^{N}\right)$ of $-\Delta u+V u=b u$ where $\xi$ is uniformly almost-periodic in the sense of Besicovich [5]. The function $\xi$ does not belong to $L^{2}\left(\Re^{N}\right)$ so we introduce a sequence of truncations. For $k \in \aleph, l e t$

$$
z_{k}(x)=k^{N / 2} \eta(x / k) \xi(x) \text { for } x \in \Re^{N}
$$

where $\eta \in C_{0}^{\infty}\left(\Re^{N}\right)$ is such that $\eta(x) \geq 0$ for all $x$ and

$$
\eta(x)=\left\{\begin{array}{l}
1 \text { for }|x| \leq 1 \\
0 \text { for }|x| \geq 2
\end{array} .\right.
$$

If follows that $z_{k} \in H^{2}\left(\Re^{N}\right) \cap C^{1}\left(\Re^{N}\right)$ and that

$$
\left((S-b I) z_{k}, z_{k}\right)=\left\langle(A-b L) z_{k}, z_{z}\right\rangle .
$$

As is shown in [29] the Riemann-Lebesgue Lemma for uniformly almostperiodic functions leads to the following properties of the sequence $\left\{z_{k}\right\}$. The mean-value of a uniformly almost-periodic function $f$ is denoted by $\mu(f)$. As $k \rightarrow \infty$ we have that 
1. $\left|z_{k}\right|_{2}^{2} \rightarrow \mu\left(\xi^{2}\right)|\eta|_{2}^{2}$

2. $k^{2}\left\langle(A-b L) z_{k}, z_{z}\right\rangle \rightarrow \mu\left(\xi^{2}\right)|\nabla \eta|_{2}^{2}$,

3. $k^{2}\left|(S-b I) z_{k}\right|_{2}^{2} \rightarrow 4 \sum_{i, j=1}^{N} \mu\left(\partial_{i} \xi \partial_{j} \xi\right) \int \partial_{i} \eta \partial_{j} \eta d x$,

4. $\left|\nabla z_{k}\right|_{2}^{2} \rightarrow \mu\left((b-V) \xi^{2}\right)|\eta|_{2}^{2}$.

It follows that $\left\{\left\|z_{k}\right\|\right\}$ is bounded and that there exists $k_{0} \in \aleph$ and $\gamma>0$ such that $\left\|z_{k}\right\| \geq \gamma$ for all $k \geq k_{0}$. Hence we can define a sequence $\left\{u_{k}\right\}$ in $H$ by setting

$$
u_{k}=\frac{z_{k}}{\left\|z_{k}\right\|} \text { for } k \geq k_{0}
$$

Using this sequence we find that the condition $\mathrm{T}(\delta)$ is satisfied in the following circumstances.

Under the hypotheses (A1), (A2), (A4) and $\mathrm{D}(\tau)$, the condition $\mathrm{T}(\delta)$ is satisfied for all $\delta<\frac{4}{N(p-2)+2 \tau}$.

If (A1), (A2), (A4) and (A7) hold, then the condition $\mathrm{T}(\delta)$ is satisfied for all $\delta<\frac{4}{N(p-2)}$.

A variety of results concerning bifurcation can be now be derived from the general theory. We shall only formulate a few typical examples and then we shall make a series of remarks about other cases which have been treated.

Theorem 6.1 Suppose that the hypotheses (A1), (A2) and (A6) are satisfied.

(I) If (A3) holds, if $D(\tau)$ is satisfied for some $\tau \in[0,2)$ and if $p<$ $2+\frac{2(2-\tau)}{N}$, then for every $r>0$, there exists a solution $\left(\lambda_{r}, u_{r}\right)$ of equation (10) with $\int u_{r}^{2} d x=r^{2}$. Furthermore, $\Lambda=0 \in B$ and there is bifurcation to the left at 0 of order $\theta$ for any $\theta<\frac{2(2-\tau)-N(p-2)}{4(p-2)}$. There is also a constant $K$ such that $-K r^{\gamma}<\lambda_{r}<0$ where $\gamma=1 /\left\{\frac{1}{p-2}-\frac{N}{4}\right\}$.

(II) If $\left\{x \in \Re^{N}: r(x)=0\right\}$ has measure zero, then for every $\lambda<\dot{\Lambda}$ there exists a solution $\left(\lambda, u_{\lambda}\right)$ of equation (10) with $u_{\lambda} \in H \backslash\{0\}$. 
This is a consequence of Theorem 11 and part (II) follows from Theorem 4.

Note that the property (A6) means that $\mathrm{D}(\tau)$ cannot be satisfied for $\tau=0$. The first results for this case were given in [40], [65], [67].

Theorem 6.2 Let $V$ satisfy the hypotheses (A1) to (A3) and (A5) with $p<$ $2+\frac{4}{N}$ in (A2). Suppose that $r=r_{1}+r_{2}$ where $r_{1}$ and $r_{2}$ have the following properties.

(a) $r_{1} \geq 0$ a.e. and $r_{1}(x)=r_{1}(y)$ whenever $|x|=|y|$.

(b) $\liminf _{|x| \rightarrow \infty} r_{1}(|x|)>0$.

(c) $r_{2} \geq 0$ a.e. and $r_{2}$ satisfies (A6).

Then $\Lambda=0 \in B$.

This follows from Corollary 16.

In the above result the assumptions (b) and (c) imply that $r$ satisfies the condition $\mathrm{D}(0)$.

There are many variants of these conditions on $r$ which can be used in the case where $V \equiv 0$. See [76], [75], [14], [16], [84] for some examples. For results concerning $p>2+\frac{4}{N}$, see [32], [58], [56], [71], [82], [81].

The following result due to Rother [57] allows the coefficient $r$ to change sign. Let $r_{+}=\max \{0, r\}$ and let $r_{-}=\max \{0,-r\}$ so that $r=r_{+}-r_{-}$.

Theorem 6.3 Suppose that $V \equiv 0$ and that $r_{+}=r_{1}+r_{2}$ where

(a) $r_{1} \in L^{\infty}\left(\Re^{N}\right), r_{1} \geq 0$ and $r_{1}$ satisfies (A6),

(b) $r_{2} \in L^{q}\left(\Re^{N}\right)$ for some $q \in\left(\frac{2 N}{4-N(p-2)}, \infty\right)$ and $r_{2} \geq 0$,

(c) $r_{-} \in L_{l o c}^{1}\left(\Re^{N}\right)$.

Suppose also that $r$ satisfies the condition $D(\tau)$ for some $\tau \in[0,2)$ and that $p<2+\frac{2(2-\tau)}{N}$.

Then for every $r>0$, there exists a solution $\left(\lambda_{r}, u_{r}\right)$ of equation (10) with $\int u_{r}^{2} d x=r^{2}$. Furthermore, $\Lambda=0 \in B$ and there is bifurcation to the left at 0 .

Now we give some results about bifurcation into spectral gaps for (10). Other variants can be found in [42], [25], [11] 
Theorem 6.4 Let the hypotheses (A1), (A2) and (A4) be satisfied. Suppose also that

either (i) (A6) holds and $D(\tau)$ is satisfied for some $\tau \in[0,2)$ such that $p<2+\frac{2(2-\tau)}{4}$,

or (ii) (A ) holds but $r \not \equiv 0$ and $p<2+\frac{4}{N}$.

Let $(a, b)$ be a maximal interval in $\Re \backslash \sigma(S)$.

Then $b \in B$ and there is bifurcation to the left at $b$ of order $\theta$ for any $\theta<\frac{2(2-\tau)-N(p-2)}{4(p-2)}$ in case (i) and $\theta<\frac{4-N(p-2)}{4(p-2)}$ in case (ii).

This follows from Theorem 17.

Theorem 6.5 Let the hypotheses (A1), (A2) and (A4) be satisfied with $p<$ $2+\frac{4}{N}$. Suppose that $r=r_{1}+r_{2}$ where

(a) $r_{1} \geq 0$ a.e. and $r_{1}$ satisfies (A7) but $r_{1} \not \equiv 0$ a.e., and

(b) $r_{2} \geq 0$ a.e. and $r_{2}$ satisfies (A6).

Let $(a, b)$ be a maximal interval in $\Re \backslash \sigma(S)$.

Then $b \in B$ and there is bifurcation to the left at $b$.

This follows from Theorem 19.

The first results concerning the bifurcation of an infinite number of branches from $\Lambda$ for the equation (2) were obtained by Ruppen [60]. A different approach was developed in [33] for radially symmetric problems where the solutions can be characterized by their nodal properties. Later the following more general result was obtained by Rother covering cases where the coefficient $r$ may be unbounded and may change sign.

Theorem 6.6 Suppose that $V \equiv 0$ and that $r$ satisfies the condition $D(\tau)$ for some $\tau>[N(p-2)-4] / 2$. Suppose also that $|r|=r_{1}+r_{2}+r_{3}$ where

(a) $r_{1} \in L^{\infty}\left(\Re^{N}\right), r_{1} \geq 0$ and $r_{1}$ satisfies (A6),

(b) $r_{2} \in L^{q}\left(\Re^{N}\right)$ for some $q \in\left(\frac{2 N}{4-N(p-2)}, \infty\right)$ and $r_{2} \geq 0$.

Then, for each $\lambda<0$, the equation (10) has infinitely many distinct pairs of solutions $\left(\lambda, \pm u_{\lambda}^{k}\right)$ with $u_{\lambda}^{k} \in H \backslash\{0\}$ for $k \in \aleph$ and $\left\|u_{\lambda}^{k}\right\| \rightarrow 0$ as $\lambda \rightarrow 0$ for all $k$.

See $[59]$.

Concerning multiple solutions in spectral gaps for (10) the first results are due to Heinz [27]. The following one due to Jeanjean [31], which can 
be derived from Theorem 21, has a slightly sharper conclusion. Further innovative work in this direction is contained in a interesting recent paper by Heinz, [28], which shows the difference between right and left end-points of spectral gaps as far as the multiplicity of solutions is concerned.

Theorem 6.7 Let the hypotheses (A1), (A2), (A4), (A6) and $D(\tau)$ be satisfied with $p<2+\frac{2(2-\tau)}{N}$. Let $(a, b)$ be a maximal interval in $\Re \backslash \sigma(S)$.

Then there exists $r_{0}>0$ such that, for every $r \in\left(0, r_{0}\right)$, there is a sequence $\left\{\left(\lambda_{r}^{k}, u_{r}^{k}\right\}_{k=1}^{\infty}\right.$ of distinct solutions of equation (10) such that $\int\left[u_{r}^{k}(x)\right]^{2} d x=r^{2}$ for all $k$. Furthermore there is a constant $K>0$ such that

$$
b-K r^{(p-2) / 2}<\lambda_{r}^{k}<b \text { and }\left\|u_{r}^{k}\right\| \leq K r \text { for all } k .
$$

Remark For the case $N=1$ and $V \equiv 0$, Ruppen [62] has used his results to establish the bifurcation at 0 of solutions having any prescribed number of zeros. Let us emphasize that while Theorem 9 allows him to deal with cases where (A6) is satisfied, he has been able to treat cases where $D(0)$ holds by using his result which we have included as Theorem 10 .

Finally we review some other aspects of the problem.

\section{$\mathrm{L}^{p}$-bifurcation}

The results presented so far in this section refer to bifurcation for (10) with respect to the usual norm in $H^{1}\left(\Re^{N}\right)$. However, at least in the case where $V \equiv 0$, necessary and sufficient conditions (which, of course, depend on $p$ ) have been found for bifurcation to the left at 0 with respect to the usual norm on $L^{p}\left(\Re^{N}\right)$ for any $p \geq 1$. See [75], [73], [74], [82], [81].

\section{Continua of solutions}

For some special situations the bifurcation of an unbounded connected set of solutions of (10) has been established by applying degree theory to a sequence of approximate problems having simple eigenvalues, followed by a passage to the limit. The best result in this direction is due to Toland who considers the Dirichlet problem for (10) on $\Omega_{k}=\left\{x \in \Re^{N}:|x|<k\right\}$ and then lets $k \rightarrow \infty$. Amongst other things he obtains the following result.

Theorem 6.8 Suppose that $V \equiv 0$ and that $2<p<2^{*}$. Let $r(x)=\rho(|x|)$ for all $x \in \Re^{N}$ where $\rho:[0, \infty) \rightarrow(0, \infty)$ is a continuous, non-increasing function with $\lim _{t \rightarrow \infty} \rho(t)>0$. 
Then there is a set $\mathcal{C}$ which has the following properties.

(i) $\mathcal{C}$ is an unbounded connected subset of $(-\infty, 0] \times L^{q}\left(\Re^{N}\right)$ for all $q \geq 1$ such that $p<2+\frac{2 q}{N}$.

(ii) $(0,0) \in \mathcal{C}$.

(iii) If $(\lambda, u) \in \mathcal{C}$, then $u$ is a positive, radially symmetric and radially decreasing solution of (10) which decays to zero as $|x| \rightarrow \infty$.

See $[82]$.

One of the first results [18] concerning bifurcation from the essential spectrum in fact yields the bifurcation of a continuum of solutions from the infimum of the essential spectrum. It deals with a one dimensional equation like (10) but the nonlinearity must contain a contribution in the form of an integral operator. Instead of studying the equation on an increasing sequence of bounded domains, we studied the problem on the whole domain but introduced a sequence of potentials $V_{k}$ converging to zero and such that for each fixed $k$ the linearized equation has negative, simple eigenvalues. Global bifurcation for the approximate equation occurs at each of these eigenvalues and, by passing to the limit, global branches of solutions for the original equation bifurcating from 0 are obtained.

The case $N=1$ is treated in [81]. For a semilinear elliptic equation on a strip, see [2].

Curves of solutions

The bifurcation of a curve of solutions from the lowest point of the essential spectrum has been established for some one dimensional problems [48], [49], [72], [77], [74] and for some problems involving some degree of radial symmetry. Under more restrictive conditions such a curve can even be extended globally [72], [64], [80].

Recently Küpper and Merziglod [39], [52] have established both local and global results showing the bifurcation of curves of solutions from the right hand end points of spectral gaps for the one dimensional nonlinear periodic Schrödinger equation. Their results require the coefficient $r$ in (10) to have compact support but nonetheless they provide valuable information about how the branches of solutions behave which should stimulate further progress in this direction. 
Quasilinear equations

The equation (10) has a semilinear structure but some results about bifurcation from the infimum of the essential spectrum for equations having a quasilinear structure have also been obtained, [45], [17], [15].

Other equations

There has been a lot of work on the case where the coefficient $r$ of the nonlinear term in (10) is negative and $V \equiv 0$. Clearly there are no solutions with $\lambda \leq 0$ and to obtain solutions with $\lambda>0$ we must suppose that the coefficient $r(x) \rightarrow-\infty$ relatively fast as $|x| \rightarrow \infty$. Consequently the equation is no longer Fréchet differentiable at the origin in the usual function spaces and so the role of the linearization is not so clear. Nontheless there is bifurcation to the right at $\lambda=0$. See [36], [37], [7], [3] for the earliest contributions in this direction and [24], [38], [70] for surveys. Some more recent developments are given in [24], [22], [23].

In the equation (10) the essential spectrum appears because the problem is posed on an unbounded domain which we have chosen to be $\Re^{N}$. However the same situation also occurs on a bounded domain if the coefficients of the linear differential operator are sufficiently singular [4]. 


\section{References}

[1] S. Agmon. Lectures on Exponential Decay of Solutions of Second-Order Elliptic Equations. Princeton University Press, Princeton, 1982.

[2] C. J. Amick and J. F. Toland. Nonlinear elliptic eigenvalue problems on an infinite strip - global theory of bifurcation and asymptotic bifurcation. Math. Ann., 262:313-342, 1983.

[3] V. Benci and D. Fortunato. Does bifurcation from the essential spectrum occur ? Comm. Partial Diff. Equats, 6:249-272, 1981.

[4] H. Berestycki and M. Esteban. Sur la structure des solutions pour un problème elliptique dégénéré. multiplicité et bifurcations. C. R. Acad. Sci. Paris, 315:1375-1380, 1992.

[5] A. S. Besicovich. Almost Periodic Functions. Cambridge University Press, Cambridge, 1932.

[6] R. Böhme. Die Losung der Verzweigungsgleichungen fur nichtlineare Eigenwertprobleme. Math. Z., 127:105-126, 1972.

[7] A. Bongers, H.-P. Heinz, and T. Küpper. Existence and bifurcation theorems for nonlinear elliptic eigenvalue problems. J. Diff. Equats, 47:327-357, 1983 .

[8] H. Brézis. Analyse Fonctionnelle. Masson, Paris, 1983.

[9] H. Brézis and L. Nirenberg. Remarks on finding critical points. Comm. Pure Appl. Math., 44:939-963, 1991.

[10] B. Buffoni. Un Problème Variationnel Fortement Indefini Sans Compacité. PhD thesis, EPFL, Lausanne, 1992.

[11] B. Buffoni and L. Jeanjean. Bifurcation from the spectrum towards regular values. J. reine angew. Math., 445:1-29, 1993. 
[12] B. Buffoni and L. Jeanjean. Minimax characterization of solutions for a semilinear elliptic equation with lack of compactness. Ann. Inst. Henri Poincare, Anal Non Lineaire, 10:377-404, 1993.

[13] B. Buffoni, L. Jeanjean, and C. A. Stuart. Existence of a nontrivial solution to a strongly indefinite semilinear equation. Proc. A.M.S., 119:179186, 1993.

[14] D. M. Cao. Positive solutions and bifurcation from the essential spectrum of a semilinear elliptic equation on $\mathrm{R}^{n}$. Nonlinear Anal. T.M.A., 15:1045-1052, 1990.

[15] D. M. Cao. Bifurcation for quasilinear elliptic equations in $\mathrm{R}^{n}$ with natural growth conditions. J. London Math. Soc., 44:514-524, 1991.

[16] D. M. Cao. Eigenvalue problems and bifurcation of semilinear elliptic equations in $\mathrm{R}^{n}$. Nonlinear Anal. T.M.A., 24:529-554, 1995.

[17] D. M. Cao, G. Li, and S. Yan. Bifurcation for quasilinear elliptic equations on $\mathrm{R}^{n}$ with natural growth conditions. Proc. Royal Soc. Edinburgh, $113 \mathrm{~A}: 215-228,1989$.

[18] R. Chiappinelli and C. A. Stuart. Bifurcation when the linearized problem has no eigenvalues. J.Diff.Equats, 30:296-307, 1978.

[19] Y. Demay. Bifurcation d'un soliton pour une équation de la physique des plasmas. C. R. Acad. Sci. Paris, 285:769-772, 1977.

[20] M. P. Eastham. The Spectral Theory of Periodic Differential Equations. Scottish Academic Press, Edinburgh, 1973.

[21] D. E. Edmunds and W. D. Evans. Spectral Theory and Differential Operators. Oxford University Press, Oxford, 1987.

[22] H.-P. Heinz. Nodal properties and bifurcation from the essential spectrum for a class of nonlinear Sturm-Liouville problems. J. Diff. Equats, 64:299-333, 1986.

[23] H.-P. Heinz. Free Ljusternik-Schnirelman theory and the bifurcation diagrams of certain singular nonlinear problems. J. Diff. Equats, 66:263$300,1987$. 
[24] H.-P. Heinz. Bifurcation from the essential spectrum and variational techniques. In Differential Equations and Applications, pages 432-440. Ohio University Press, Athens, Ohio, 1989.

[25] H.-P. Heinz. Bifurcation from the essential spectrum and nonlinear perturbations of Hill's equation. In Differential Equations : Stability and Control, pages 219-226. Marcel Dekker, New York, 1990.

[26] H.-P. Heinz. Lacunary bifurcation for operator equations and nonlinear boundary value problems on $\mathrm{R}^{n}$. Proc. Royal Soc. Edinburgh, 118A:237$270,1991$.

[27] H.-P. Heinz. Existence and gap-bifurcation of multiple solutions to certain nonlinear eigenvalue problems. Nonlinear Anal. T.M.A., 21:457484, 1993.

[28] H.-P. Heinz. On the number of solutions of nonlinear Schrödinger equations and on unique continuation. J. Diff. Equats, 116:149-171, 1995.

[29] H.-P. Heinz, T. Küpper, and C. A. Stuart. Existence and bifurcation of solutions for nonlinear perturbations of the periodic Schrödinger equation. J. Diff. Equats, 100:341-354, 1992.

[30] J. Ize. Topological bifurcation. In Topological Analysis : Degree, Singularity and Variations, pages 341-463. Birkhauser, Basel, 1995.

[31] L. Jeanjean. Approche Minimax des Solutions d'une Equation Semilineaire Elliptique en Absence de Compacité. PhD thesis, EPFL, Lausanne, 1992.

[32] L. Jeanjean. Existence of solutions with prescribed norm for semilinear elliptic equations. Nonlinear Anal. T.M.A., to appear.

[33] C. K. R. T. Jones and T. Küpper. Characterisation of bifurcation from the continuous spectrum by nodal properties. J. Diff. Equats, 54:196$220,1984$.

[34] T. Kato. Perturbation Theory for Linear Operators. Springer, Berlin, 1966.

[35] M. A. Krasnoselski. Topological Methods in the Theory of Nonlinear Integral Equations. Pergammon, London, 1964. 
[36] T. Küpper. The lowest point of the continuous spectrum as a bifurcation point. J. Diff. Equats, 34:212-217, 1979.

[37] T. Küpper. On minimal nonlinearities which permit bifurcation from the continuous spectrum. Math. Methods Appl. Sci., 1:572-580, 1979.

[38] T. Küpper. Verzweigungen aus dem wesenlichten Spektrum. GAMMMitteilungen, 1, pages 11-22, 1991.

[39] T. Küpper and T. Mrziglod. On the bifurcation structure of nonlinear perturbations of Hill's equation at boundary points of the spectrum. SIAM J. Math. Anal., 1995.

[40] T. Küpper and D. Riemer. Necessary and sufficient conditions for bifurcation from the continuous spectrum. Nonlinear Anal. T.M.A., 3:555$561,1979$.

[41] T. Küpper and C. A. Stuart. Bifurcation into gaps in the essential spectrum. J. reine angew. Math., 409:1-34, 1990.

[42] T. Küpper and C. A. Stuart. Gap bifurcation for nonlinear perturbations of Hill's equation. J. reine angew. Math., 410:23-52, 1990.

[43] T. Küpper and C. A. Stuart. Bifurcation at boundary points of the continuous spectrum. In Nonlinear Diffusion Equations and Their Equilibrium States, 3, Proc. Of Gregynogg Conf. 1989, pages 287-297. Birkhauser, Basel, 1992.

[44] T. Küpper and C. A. Stuart. Necessary and sufficient conditions for gap bifurcation. Nonlinear Anal., 18:893-903, 1992.

[45] T. Küpper and J. Weyer. Maximal monotonicity and bifurcation from the continuous spectrum. Nonlinear Anal. T.M.A., 7:723-731, 1982.

[46] L. Liu. Bifurcation for potential operators. Nonlinear Anal. T.M.A., 15:345-353, 1990.

[47] L. A. Ljusternik and V. J. Sobolev. Elements of Functional Analysis. Hindustan Publishing Corp., Delhi, 1974. 
[48] R. J. Magnus. On the asymptotic properties of solutions to a differential equation in the case of bifurcation without eigenvalues. Proc. Royal Soc. Edinburgh, 104:137-160, 1986.

[49] R. J. Magnus. On perturbations of a translationally invariant differential equation. Proc. Roy. Soc. Edinburgh, 110:1-25, 1988.

[50] A. Marino. La biforcazione nel caso variazionale. Conf. Sem. Math. Uni. Bari, 132, 1972.

[51] J. Mawhin and M. Willem. Critical Point Theory and Hamiltonian Systems. Springer, Berlin, 1989.

[52] T. Mrziglod. Unbounded solution components for nonlinear Hill's equations. Proc. Royal Soc. Edinburgh, 125:1131-1169, 1995.

[53] P. H. Rabinowitz. Variational methods for nonlinear eigenvalue problems. In C.I.M.E. Varenna, 1974.

[54] M. Reed and B. Simon. Methods of Modern Mathematical Physics. Academic Press, New York, 1978.

[55] M. Robert and C. A. Stuart. Intrinsic structure of the critical liquid-gas interface. Phys. Rev. Letters, 49:1434-1437, 1982.

[56] W. Rother. Bifurcation for a semilinear elliptic equation on $\mathrm{R}^{n}$ with radially symmetric coefficients. Manuscripta Math., 65:413-425, 1989.

[57] W. Rother. Bifurcation of nonlinear elliptic equations on $\mathrm{R}^{n}$. Bull. London Math. Soc., 21:567-572, 1989.

[58] W. Rother. Existence and bifurcation results for a class of nonlinear boundary value problems in (0,oo). Comment. Math. Univ. Carolinae, 32:297-305, 1991.

[59] W. Rother. The existence of infinitely many solutions all bifurcating from l=0. Proc. Royal Soc.Edinburgh, 118A:295-303, 1991.

[60] H.-J. Ruppen. The existence of infinitely many bifurcating branches. Proc. Royal Soc. Edinburgh, 101A:307-320, 1985. 
[61] H.-J. Ruppen. Inherited bifurcation. Nonlinear Anal. T.M.A., 19:993$1000,1992$.

[62] H.--J. Ruppen. Nodal characterization of bifurcating branches in $\mathrm{L}^{p}(\mathrm{R})$ for a semilinear equation. J. Diff. Equats, 99:153-203, 1992.

[63] M. Struwe. Variational Methods. Springer, Berlin, 1990.

[64] C. A. Stuart. Des bifurcations sans valeurs propres. C. R. Acad. Sci. Paris, 284:1373-1375, 1977.

[65] C. A. Stuart. Bifurcation pour des problèmes de Dirichlet et de Neumann sans valeurs propres. C. R. Acad. Sci. Paris, 288:761-764, 1979.

[66] C. A. Stuart. An introduction to bifurcation theory based on differential calculus. In R. J. Knops, editor, Nonlinear Analysis : Heriot-Watt Symposium, Vol IV, pages 76-135. Pitman, 1979.

[67] C. A. Stuart. Bifurcation for Neumann problems without eigenvalues. J. Diff. Equats, 36:391-407, 1980.

[68] C. A. Stuart. Bifurcation for variational problems when the linearisation has no eigenvalues. J. Functional Anal., 38:169-187, 1980.

[69] C. A. Stuart. Bifurcation for Dirichlet problems without eigenvalues. Proc. London Math. Soc., 45:169-192, 1982.

[70] C. A. Stuart. Bifurcation from the essential spectrum. In H. W. Knobloch and K. Schmitt, editors, Lecture Notes in Mathematics No 1017, Lecture Notes in Mathematics No 1017, pages 573-596. Springer, 1982.

[71] C. A. Stuart. A variational aproach to bifurcation in $\mathrm{L}^{p}$ on an unbounded symmetrical domain. Math. Ann., 263:51-59, 1983.

[72] C. A. Stuart. A global branch of solutions to a semilinear equation on an unbounded interval. Proc. Royal Soc. Edinburgh, 101A:273-282, 1985.

[73] C. A. Stuart. Bifurcation in $\mathrm{L}^{p}(\mathrm{R})$ for a semilinear equation. J. Diff. Equats, 64:294-316, 1986. 
[74] C. A. Stuart. Bifurcation from the continuous spectrum in $\mathrm{L}^{p}(\mathrm{R})$. In Bifurcation: Analysis, Algorithms, Applications, pages 306-318. Birkhauser, Basel, 1987.

[75] C. A. Stuart. Bifurcation in $\mathrm{L}^{p}\left(\mathrm{R}^{n}\right)$ for a semilinear elliptic equation. Proc. London Math. Soc., 57:511-541, 1988.

[76] C. A. Stuart. Bifurcation from the essential spectrum for some noncompact nonlinearities. Math. Meth. Appl. Sci., 11:525-542, 1989.

[77] C. A. Stuart. Bifurcation of homoclinic orbits and bifurcation from the essential spectrum. SIAM J. Math. Anal., 20:1145-1171, 1989.

[78] C. A. Stuart. Self-trapping of an electromagnetic field and bifurcation from the essential spectrum. Arch. Rational Mech. Anal., 113:65-96, 1991.

[79] C. A. Stuart. Bifurcation into spectral gaps. Supplement to Bull. Soc. Math. Belgique, 1995.

[80] A. Tertikas. Global bifurcation analysis and uniqueness for a semilinear problem. Proc. Royal Soc. Edinburgh, 111A:265-284, 1989.

[81] J. F. Toland. Global bifurcation for Neumann problems without eigenvalues. J. Diff. Equats, 44:82-110, 1982.

[82] J. F. Toland. Positive solutions of nonlinear elliptic equations-existence and nonexistence of solutions with radial symmetry. Trans. A.M.S., 282:335-354, 1984.

[83] M. Willem. Minimax Theorems. Springer, Berlin, to appear.

[84] H.-S. Zhou and X.-P. Zhu. Bifurcation from the essential spectrum of superlinear elliptic equations. Applic. Anal., 28:51-66, 1988. 